\title{
A Review on Plants Used for Improvement of Sexual Performance and Virility
}

\author{
Nagendra Singh Chauhan, ${ }^{1,2}$ Vikas Sharma, ${ }^{1}$ V. K. Dixit, ${ }^{1}$ and Mayank Thakur ${ }^{1,3}$ \\ ${ }^{1}$ Department of Pharmaceutical Sciences, Dr. Harisingh Gour Central University, Sagar, Madhya Pradesh 470003, India \\ ${ }^{2}$ Drugs Testing Laboratory Avam Anusandhan Kendra, GE Road, Raipur, Chhattisgarh 492010, India \\ ${ }^{3}$ Institute for Laboratory Medicine Clinical Chemistry, and Pathobiochemistry, Charite Universitätsmedizin, \\ Campus Virchow Klinikum, Augustenburger Platz 1, 12200 Berlin, Germany
}

Correspondence should be addressed to Nagendra Singh Chauhan; chauhan.nagendra@gmail.com and Mayank Thakur; mayankthakur25@gmail.com

Received 28 February 2014; Revised 13 July 2014; Accepted 24 July 2014; Published 18 August 2014

Academic Editor: Kazem M. Azadzoi

Copyright (c) 2014 Nagendra Singh Chauhan et al. This is an open access article distributed under the Creative Commons Attribution License, which permits unrestricted use, distribution, and reproduction in any medium, provided the original work is properly cited.

\begin{abstract}
The use of plant or plant-based products to stimulate sexual desire and to enhance performance and enjoyment is almost as old as the human race itself. The present paper reviews the active, natural principles, and crude extracts of plants, which have been useful in sexual disorders, have potential for improving sexual behaviour and performance, and are helpful in spermatogenesis and reproduction. Review of refereed journals and scientific literature available in electronic databases and traditional literature available in India was extensively performed. The work reviews correlation of the evidence with traditional claims, elucidation, and evaluation of a plausible concept governing the usage of plants as aphrodisiac in total. Phytoconstituents with known structures have been classified in appropriate chemical groups and the active crude extracts have been tabulated. Data on their pharmacological activity, mechanism of action, and toxicity are reported. The present review provides an overview of the herbs and their active molecule with claims for improvement of sexual behaviour. A number of herbal drugs have been validated for their effect on sexual behavior and fertility and can therefore serve as basis for the identification of new chemical leads useful in sexual and erectile dysfunction.
\end{abstract}

\section{Introduction}

Male reproductive capacity was found to be deficient in nearly $50 \%$ of infertile couples according to a study carried out by the World Health Organization in 1987. Although further figures for this decade are still awaited, it is certain that stressful life style has enhanced the number of subject's suffering from one form of sexual dysfunction or the other. Main factors that decrease the probability of conception in the female partner are frequently congenital, immunological, iatrogenic, or endocrine cause. Oligozoospermia, sexual, and ejaculatory dysfunction are further responsible for inability to conceive in numerous cases [1]. Although many synthetic drugs are available and/or used to treat these problems, some of the drawbacks for these drugs include them being expensive and also their ability to provoke serious adverse effects, effective natural treatments are therefore still in demand. Even if many of the plants or natural products claim to prove their effectiveness without scientific evidence, a number of them are active and possess biological activity, proven by scientific data. Moreover, there is a dearth of systematic review of scientific literature on experimental evidence generated for medicinal plants useful in treating erectile dysfunction and there is a need for in depth pharmacological evaluation [2].

Advancement in the understanding of pharmacological basis of erectile and sexual functions at molecular levels is turning out to be stepping stones towards isolating the crucial physiologic factors involved in sexual arousal, thus helping to narrow down the search for aphrodisiac substances of choice. Many people do not believe in love potions or aphrodisiacs, but countless numbers of men and women have used them down through the centuries, and there is clear proof that they 


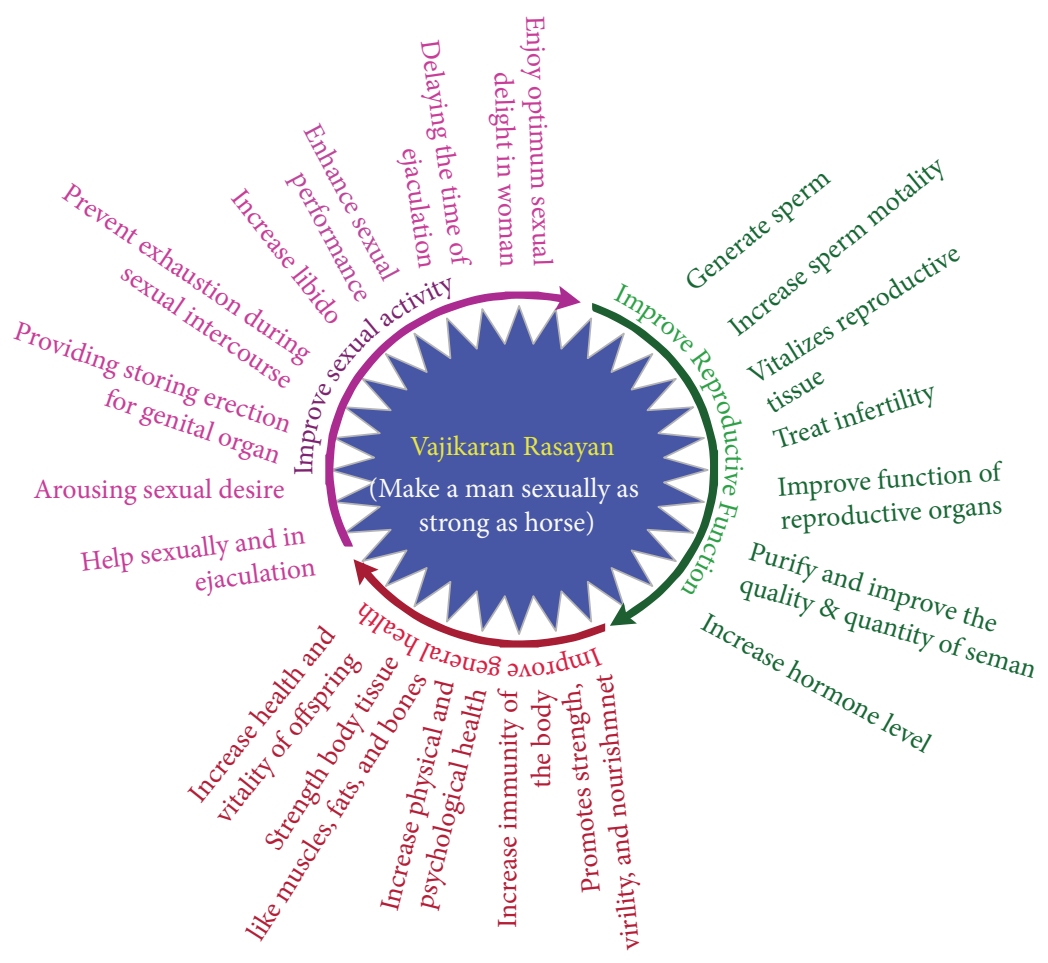

FIgURE 1: Action of Vajikaran Rasayana.

are still in use today. The skepticism towards the concept of aphrodisiac is not unjustified, although a systematic evaluation and compilation of scientific information may provide a basis for the evidence-based utilization of herbal drugs for treatment of sexual dysfunction in general. The present review is an attempt to consummate the available scientific information on various herbal drugs, which have been evaluated for their effect on sexual performance and functionality. The review also includes known evidences collected for the involvement of herbal drugs on neural, nitric oxide and hormone-dependent mechanisms and their role on sexual functions. A number of plants have been discussed in detail and a few others are only tabulated; a major criterion for this arrangement was the ethnopharmacological relevance of the plant in the Ayurvedic system of medicine. Nonetheless, it is very important to mention that this does not entail a grading system for the plants described in the paper and some of the plants only listed in tabular form may also be of high scientific relevance.

\section{Historical Background}

The word "Aphrodisiac" is derived from "Aphrodite" the Greek goddess of love. By definition aphrodisiacs are the substance, which stimulate sexual desire (Greek-Aphrodisiakossexual) [3]. A variety of plants have been used as sex stimulants or sexual performance enhancer in traditional systems of medicine of various countries [4-6]. Practitioners of Ayurveda the traditional system of medicine in India recognized the vital importance of virility and formulated
Vajikarna therapy [7] (Table 1). Modern day concept for the term "aphrodisiac" can be considered close to the Vajikarna concept defined in traditional texts of Ayurvedic medicine.

\section{Vajikaran in Ayurvedic Texts}

Vajikaran as a concept has been defined in the Rig Veda and the Yajurveda, the first written texts of medicine, in Ayurveda. Vajikarana herbs are also the basis for therapies recommended in Kamasutra, a treatise defining methods for appropriate sexual satisfaction amongst couples. An excerpt of the definition derived from these texts suggests that a youth in sound health taking regularly some sort of Vajikarana remedy may enjoy the pleasure of youth every night during all the seasons of the year [8]. Old men, wishing to enjoy sexual pleasure or to secure the affections of women, as well as those suffering from senile decay or sexual incapacity, and persons weakened with sexual excesses may also use Vajikaran remedies. They are highly beneficial to handsome and opulent youths and to persons who have got many wives. According to Rasendra Sara Sangrah an ayurvedic text Vajikaran remedy makes a man sexually as strong as a horse (Vaji) and enables him to cheerfully satisfy the heat and amorous ardours of young maidens (Figure 1) $[9,10]$. Though in scientific terms these claims may represent a populous outlook, the popularity of Vajikaran in Ayurvedic system of medicine is nonetheless undisputed with numerous claims and textual references made to them during the course of human history. 
TABLE 1: List of plant reported in Ayurveda as Vajikaran Rasayan.

\begin{tabular}{|c|c|c|c|c|c|}
\hline $\begin{array}{l}\text { Serial } \\
\text { number }\end{array}$ & Hindi name & Botanical name & Family & Parts use & Uses \\
\hline 1 & Akarkara & Anacyclus pyrethrum DC & Asteraceae & Dried roots & $\begin{array}{l}\text { Vajikaran, } \\
\text { Balakarka }\end{array}$ \\
\hline 2 & Akharot & Juglans regia Linn. & Juglandaceae & Dried cotylcdous & Vrsya, Bala, Sukral \\
\hline 3 & Adarakha & Zingiber officinalis Rosc. & Zingiberaceae & Fresh rhizomes & Vrsya \\
\hline 4 & Bhrngaraja & Elcipta alba nassle & Asteraceae & Whole plant & $\begin{array}{l}\text { Balya, } \\
\text { Rasayana }\end{array}$ \\
\hline 5 & Manduka parni & Bacopa monnieri Linn. & Scrophularaceae & Dried whole plant & Rasayana \\
\hline 6 & Anar & Punica granatum Linn. & Punicaceae & Dried seed & $\begin{array}{l}\text { Sukralya, } \\
\text { Balya }\end{array}$ \\
\hline 7 & Gambhari & Gmeline arborea Roxb. & Verbenaceae & Dried fruit & $\begin{array}{l}\text { Rasayana, } \\
\text { Sukrala }\end{array}$ \\
\hline 8 & Ganna & $\begin{array}{l}\text { Saccharum officinarum } \\
\text { Linn. }\end{array}$ & Podceae & Dried stem & Vrsya, Balya \\
\hline 9 & Jayata & Sesbana sesbanl Linn. & Fabaceae & Fresh \& dried root & Rasayana \\
\hline 10 & Talmakhana & $\begin{array}{l}\text { Asteracantha longifolia } \\
\text { Nees }\end{array}$ & Acanthaceae & Whole plant seed & $\begin{array}{l}\text { Baiya, Vrsya, } \\
\text { Vajikarna }\end{array}$ \\
\hline 11 & Makoya & Solanum nigrum Linn. & Solanaceae & Dried whole plant & $\begin{array}{l}\text { Rasayana, } \\
\text { Vrsya }\end{array}$ \\
\hline 12 & Kaitha & Feronia limonia Linn. & Rutaceae & $\begin{array}{l}\text { Dried pulp of mature } \\
\text { fruit }\end{array}$ & Vrsya \\
\hline 13 & Mahuwa & Madhuca indica & Saptoceae & Flower & $\begin{array}{l}\text { Sukrala, } \\
\text { Balya }\end{array}$ \\
\hline 14 & Tesu & Butea monosperma Lam. & Fabaceae & Dried stem bark & Vrsya \\
\hline 15 & Gandha prasarini & Paederia foetida Linn. & Rubiaceae & Whole plant & Vrsya \\
\hline 16 & Piyal & $\begin{array}{l}\text { Buchanania lanzan } \\
\text { Spreng }\end{array}$ & Anacardiaceae & Seed & Vrsya, Bala \\
\hline 17 & Chaval & Oryza sativa Linn. & Poaceae & Dried root & $\begin{array}{l}\text { Balya, } \\
\text { Rasayana }\end{array}$ \\
\hline 18 & Shankhapusphi & $\begin{array}{l}\text { Convolvulus pluricaulis } \\
\text { Chois }\end{array}$ & Convolulacea & Whole plant & $\begin{array}{l}\text { Balya, } \\
\text { Rasayana }\end{array}$ \\
\hline 19 & Vidari kanda & Pueraria tuberosa DC & Leguminosae & $\begin{array}{l}\text { Sliced \& dried pieces of } \\
\text { tuberous root }\end{array}$ & $\begin{array}{l}\text { Sukralya, } \\
\text { Balya, } \\
\text { Rasayana }\end{array}$ \\
\hline 20 & Basanaay & Aconitum Chasmanthum & Ranunculaceae & Dried roots & Rasayana \\
\hline 21 & Jav & Hordeum vulgare Linn. & Poaceceae & Dried fruit & Vrsya, Balya \\
\hline 22 & Amla & Emblica officinalis & Euphorbiaceae & Fresh fruit pulp & Vrsya, Rasayna \\
\hline 23 & Vijayasara & $\begin{array}{l}\text { Pterocarpus marsupium } \\
\text { Roxb. }\end{array}$ & Leguminosae & Heart wood & Rasayna \\
\hline 24 & Asagandha & $\begin{array}{l}\text { Withania somnifera } \\
\text { Dunal }\end{array}$ & Solanaceae & Dried mature roots & $\begin{array}{l}\text { Vajikarana, Balya, } \\
\text { Rasayana }\end{array}$ \\
\hline 25 & Kunghi & Abutilon indicum Linn. & Malvaceae & Roots & Balya, Vrsya \\
\hline 26 & Bela & Aegle marmelos & Rutaceaeae & Ripe fruit & Balya \\
\hline 27 & Gokhru & Tribulus terrestris Linn. & Zygophyllaceae & Root fruit & Vrsya \\
\hline 28 & Giloe & Tinospora Cordifolia & Menispermaceae & Stem & Balya, Rasayana \\
\hline 29 & Gugal & Commiphora wightii & Burseraceae & Exudate & Balya \\
\hline 30 & Harad & Terminallia chebula Retz & Combretaceae & Mature fruit & Rasayana \\
\hline 31 & Jaiphal & Myristica fragraus & Myristicaceae & Dried seeds & Vrsya \\
\hline 32 & Kapasa & $\begin{array}{l}\text { Gossypium herbaceum } \\
\text { Linn. }\end{array}$ & Malvaceae & Seed & Vrsya \\
\hline 33 & Kasesu & Scirpus kysoor Roxb. & Cyperaceae & Rhizome & Sukra \\
\hline 34 & Kerada & $\begin{array}{l}\text { Pandanus tectorius } \\
\text { sokmel }\end{array}$ & Pandanaceae & Root & Balya, Rasayana \\
\hline
\end{tabular}


TABLE 1: Continued.

\begin{tabular}{|c|c|c|c|c|c|}
\hline $\begin{array}{l}\text { Serial } \\
\text { number }\end{array}$ & Hindi name & Botanical name & Family & Parts use & Uses \\
\hline 35 & Saunt & Foeniculum Vulgare Mill & Umbelliferae & Ripe fruit & Balya \\
\hline 36 & Bhaang & Cannabis sativa Linn. & Cannabaceae & Dried leaves & Vajikara \\
\hline 37 & Mulethi & Glycyrrhiza glabra Linn. & Leguminosae & Root & Balya, Vrsya \\
\hline 38 & Hadjod & $\begin{array}{l}\text { Cissus quadrangularis } \\
\text { Linn. }\end{array}$ & Vitaceae & Dried stem & Vrsya \\
\hline 39 & Kewandr & Mucuna prurita Hook. & Fabaceae & Mature seed & Balya, Vrsya \\
\hline 40 & Munkka & Vitis vinifera Linn. & Vitaceae & Dried mature fruit & Vrsya \\
\hline 41 & Evana & Ricinus communis Linn. & Euphorbiaceae & Fresh leaf & Vrsya \\
\hline 42 & Bichu hathjori & Martynia annua Linn. & Martyniaceae & Dried seed & Rasayan \\
\hline 43 & Kakoli & $\begin{array}{l}\text { Lillum polyphyllum } \\
\text { D.Don }\end{array}$ & Liliaceae & Tuberous root & Sukrala \\
\hline 44 & Kamal kand & Nelumbo nucifera Gaertn & Nymphaeaceae & Rhizome & Vrsya \\
\hline 45 & Kasa & $\begin{array}{l}\text { Saccharum spontaneum } \\
\text { Linn. }\end{array}$ & Poaceae & Root stock & Vrsya, Bala \\
\hline 46 & Kui & Nymphaea alba Linn. & Nymphaeaceae & Dried flowers & Balya \\
\hline 47 & Lahasun & Allium sativum Linn. & Liliaceae & Bulb & Balya, Vrsya, Rasayna \\
\hline 48 & Pitabala & Sida rhomifolia Linn. & Malvaceae & Dried root & $\begin{array}{l}\text { Sukra, } \\
\text { Balya }\end{array}$ \\
\hline 49 & Manjitha & Rubia cordifolia Linn. & Rubiaceae & Stem & Vrsya, Rasanyana \\
\hline 50 & Mashvan & Teramnus iabialis Spreny & Fabaceae & Whole plant & Balya, Vrsya \\
\hline 51 & Masur & Lens culinaris medic & Fabaceae & Dried seeds & Balya \\
\hline 52 & Pan & Piper betle Linn. & Piperaceae & Leaf & Balya, Vrsya \\
\hline 53 & Nariyal & Cocos nucifera Linn. & Arecaceae & Dried endosperm & Balya, Vrsya \\
\hline 54 & Rakta chandana & $\begin{array}{l}\text { Petrocarpus santalinus } \\
\text { Linn. }\end{array}$ & Fabaceae & Heard wood & Balya, Vrsya \\
\hline 55 & Sarivan & $\begin{array}{l}\text { Desmodium gangetium } \\
\text { DC }\end{array}$ & Fabaceae & Dried root & Balya, Vrsya \\
\hline 56 & Chaval & Oryza sativa Linn. & Poaceae & Dried fruit & Vrsya \\
\hline 57 & Sarkand & $\begin{array}{l}\text { Saccharum bengalense } \\
\text { Retz. }\end{array}$ & Poaceae & Root & Balya, Vrsya \\
\hline 58 & Gulab & Rosa centifolia Linn. & Rosaceae & Dried flower & Sukra \\
\hline 59 & Seesam & Dalbergia sissoo Roxb. & Fabaceae & Stem bark & Balya \\
\hline 60 & Jhuner & Taxus baccata Linn. & Taxaceae & Dried leaf & Sukravarahake \\
\hline 61 & Safed chandan & Santalum album Linn. & Santalaceae & Heart wood & Vrsya \\
\hline 62 & Tal & Borassus flabellifer Linn. & Araceae & Male inflorescence & Vrsya \\
\hline 63 & Louki & Lagenaria siceraria & Cucurbitaceae & Fresh fruit & Vrsya \\
\hline 64 & Neel kanal & Nymphaea stellata Willd & Nymphaeaceae & Dried flower & Rasayana \\
\hline
\end{tabular}

The meanings of the Sanskrit words are Vrsya: increase sexual potential; Rasayan: that nourishes body, boosts immunity, and helps to keep the body and mind in best of health. Balya: that acts as vitilizer; Sukra: increase sperm count.

\section{Sexual Functions: An Ayurvedic Overview}

The sexual inadequacies discussed in Ayurveda are of the following six types:

(1) A cessation of the sexual desire owing to the rising of bitter thoughts of recollection in the mind of a man, or a forced intercourse with a disagreeable woman (who fails to sufficiently rouse up the sexual desire in the heart of her mate) illustrates an instance of mental impotency.
(2) Excessive use of articles of pungent, acid, or saline taste, or of heat making articles of fare leads to the loss of the Saumya Dhatu (watery principle) of the organism. This is another kind of impotency.

(3) Virile impotency resulting from the loss of semen in persons addicted to excessive sexual pleasure without using any aphrodisiac remedy is the merit form of the virile impotency.

(4) A long-standing disease of the male generative organ (syphilis, etc.) or the destruction of a local Marma 
such as the spermatic cord destroys the power of coition altogether.

(5) Sexual incapacity from the very birth is called the congenital (Sahaja) impotency.

(6) Voluntary suppression of the sexual desire by a strong man observing perfect continence or through utter apathy produces a hardness of the spermatic fluid and is the cause of the sixth form of virile impotence.

Of the six foregoing types of impotency, the congenital form as well as the one due to the destruction of any local Marma (spermatic cord) should be regarded as incurable, the rest being curable and amenable to the measures and remedies antidotal to their respective originating causes [11].

\section{Ayurveda and the Concept of Aphrodisiacs}

Traditional Ayurvedic treatise classified aphrodisiac in the following five categories, a few plants have been provided as references for each kind of the therapeutic class defined [3].

(i) Drugs which increase the quantity of semen or stimulate the production of semen for example, Microstylis wallichii, Roscoea procera, Polygonatum verticillatum, Mucuna pruriens, and Asparagus racemosus.

(ii) Drugs which purify and improve the quality of semen for example, Saussurea lappa, Myrica nagi, Sesamum indicum, Vetiveria zizanioides, and Anthocephalus cadamba.

(iii) Drugs which improve ejaculatory functions for example, Strychnos nux vomica, Cannabis sativa, Myristica fragrans, and Cassia occidentalis.

(iv) Drugs delaying the time of ejaculation or improving ejaculatory performance for example, Sida cordifolia, Asparagus racemosus, Cinnamomum tamala, Anacyclus pyrethrum, Mucuna pruriens, and Cannabis sativum.

(v) Drugs arousing sexual desire, namely, Withania somnifera, Asparagus racemosus, Datura stramonium, Anacyclus pyrethrum, Hibiscus abelmoschus, and Opium.

Having discussed the Ayurvedic basis for the role of Vajikarana herbs, it is important to understand the role of modern pharmacology and an insight into the control of the sexual behavior in the human body.

\section{Mechanism of Sexual Behavior: Modern Perspective}

Our understanding of the process and initiation of sexual arousal is finding a more lucid basis, which stems from evidences in both preclinical and clinical studies. Sexual arousal is dependent on neural (sensory and cognitive), hormonal, and genetic factors, something also defined in Ayurveda as well but using a scientific language pertinent to this age.

\section{Brain and Neurochemical Basis of Sexual Behavior}

Drugs affecting sexuality can either act on the central nervous system (Brain) and/or on the peripheral nervous system. Drugs affecting the brain and presumably sex centers are generally attributed with an increase or decrease in sexual arousal. Drugs that affect peripheral nerves will not affect arousal directly but may affect sexual function. In some cases, drugs action is direct and involves chemical alteration of the neurons, which governs sexual arousal or function. Alternatively, some drugs may act indirectly by altering blood flow to the genitalia. Most hypotheses concerning the neurochemical basis of sexual behavior are derived from studies in animals, but in some cases support has been provided by clinical studies. Five major neurochemically distinct systems are supposed to work together for increasing sexual arousal. The transmitters include norepinephrine, dopamine, serotonin, acetylcholine, and histamine [12]. The most widely endorsed hypotheses suggest that both serotonin and dopamine are involved in the neurochemical control of sexual behavior with serotonin playing an inhibitory role and dopamine an excitatory role. Dopamine plays a crucial role in the central control of sexual behavior in males [13]. Increase in the activity of central dopaminergic systems correlates with sexual activity [14]. In vivo microdialysis in conscious male rats revealed that dopamine transmission increases sharply in the striatum, nucleus accumbens, and medial preoptic area during copulation [15-17]. This change in central neurotransmission may be permissive to a series of motor responses including penile erection. It may also modulate the activity of brain nuclei directly involved in the control of penile erection [18]. For example, drugs such as levodopa, which increase levels of dopamine in the brain, tend to be associated with increase libido and enhanced sexual function in patients suffering from abnormal dopamine activity such as that associated with Parkinson's disease. In contrast, drugs blocking dopamine function such as haloperidol cause loss of sexual arousal. It has been long suspected that monoamines play a crucial role in the regulation of sexual behaviour, particularly that of dopaminergic transmission which is facilitatory to masculine activity and both dopaminergic and adrenergic receptors are involved. Yohimbine, bromocriptine, and reserpine are alpha-adrenergic receptor blocking agents whereas yohimbine, bromocriptine, amphetamine, and apomorphine all comet with the neurotransmitter dopamine for binding to membrane sites [19]. Furthermore, some studies have also suggested that the dopamine release is also increased during sexual activity in the paraventricular nucleus of the hypothalamus and that in this hypothalamic nucleus dopamine facilitates penile erection and sexual behaviour by activating NO production in the cell bodies of oxytocin neurons controlling penile erection and sexual motivation, which project to extrahypothalamic brain areas and to the spinal cord [13, 20-24]. Therefore, there appears to be a lot of cross talk at different neuronal levels between dopamine and nitric oxide; this has been discussed further in the next section. 


\section{Nitric Oxide-Based Mechanism of Sexual Behavior}

Nitric oxide (NO) is an atypical regulatory molecule having the dual role as a secondary messenger/neurotransmitter. It has been implicated in diverse physiological functions [22]. Findings so far indicate that NO may also be a major neuronal messenger [23]. In particular, it is an established physiological mediator of penile erection [24] and in the brain; NO synthase is highly concentrated in structures directly or indirectly involved in sexual behavior (olfactory bulb, supraoptic and paraventricular nuclei, amygdala, septal structures, etc.) [25].

Recent studies suggest that NO is a major physiological stimulus for relaxation of penile vasculature and trabecular smooth muscle, essential for penile erection [26]. Relaxation of the trabecular smooth muscle of the corpus cavernosa leads to a decreased vascular resistance and increased blood flow to the penis. Alongside the increased flow, venous outflow is reduced by the compression of the subtunical venules. The combination of increased inflow and decreased outflow causes penile engorgement and erection. NO from the vascular endothelium of the sinusoids and from the nonadrenergic, noncholinergic, and cavernosal nerves appears to mediate the vasodilatation $[27,28]$. The new drug used for the treatment of erectile dysfunction, and sildenafil acts by potentiating the effect of NO by inhibiting the specific enzyme phosphodiesterase- $\mathrm{V}$ that terminates the action of NO generated cGMP in the penile vasculature [29]. Many medicinal herbs and drugs derived from these herbs have been shown to have effects on the NO signaling pathway. For example, the saponins from ginseng (ginsenosides) have been shown to relax blood vessels (probably contributing to the antifatigue and blood pressure-lowering effects of ginseng) and corpus cavernosum (thus, for the treatment of men suffering from erectile dysfunction; however, the legendary aphrodisiac effect of ginseng may be an overstatement) [30].

\section{Androgen-Based Mechanism of Sexual Behavior}

Androgens play a crucial role in the development of secondary male sexual organs such as the epididymis, vas deferens, seminal vesicle, prostate, and the penis. Furthermore, androgens are needed for puberty, male fertility, and male sexual function [29]. Testosterone is the principal androgen secreted by the testes. Testosterone is synthesized in the Leydig cells of the testes, stimulated by luteinizing hormone (LH). One of the principal effects of testosterone within the testes is the stimulation of spermatogenesis in seminiferous tubules. The testosterone- or dihydrotestosterone-receptor complex next crosses the nuclear membrane, binds to DNA, and stimulates new mRNA synthesis and, thereby, new protein synthesis. The effect of testosterone on libido may require conversion of testosterone to estradiol in the hypothalamus. The mechanisms whereby testosterone affects muscle, bone, and the erythron do not appear to require prior molecular conversion [30].
Drugs used to treat various sexual problems are found to modify the action of neurotransmitters which could be facilitatory, inhibitory, or both. Androgens are known to influence NO production in the brain as well as in the periphery [31, 32]. NO is synthesized by the enzyme nitric oxide synthase (NOS) which plays an important role in many brain functions. NO function as a neurotransmitter and NOS is present in the regions of the brain that regulate sexual functions [33]. Interestingly, administration of testosterone to castrated male rats increases the number of NO synthaselabelled neurons in the $\mathrm{mPOA}$, indicating an increase in NO synthesis [34]. NO is capable of stimulating dopamine (DA) release in the $\mathrm{mPOA}$, which in turn stimulates penile erection. This mechanism may constitute one way in which androgens stimulate sexual arousal [35].

\section{A Few Medicinal Herbs with Validated Effects on Sexual Functions}

In the present section we would discuss a few of the many well-tested Ayurvedic and other traditional herbs, which have a long standing reputation as a cure for sexual dysfunction and which have been used in numerous preparations for improving sexual performance and fertility especially in case of males. Apart from these herbs large numbers of plants have also been tested and evaluated for effect on sexual functions and reproductive parameters, a comprehensive description and names of these herbs are provided in Table 2. Many researchers have investigated the active bioconstituent present in different herbs that are responsible for enhancing sexual activity, spermatogenesis and showing other positive effect in reproductive parameters (Table 3 ).

10.1. Butea superba. Butea superba Roxb (Leguminosae) is commonly found in Thai deciduous forests and has the domestic name of "Red Kwao Krua." The plant tubers have long been consumed as a traditional medicine for the promotion of male sexual vigor. B. superba alcoholic extract $(0.01,0.1$ or $1.0 \mathrm{mg} / \mathrm{kg}$ BW/day) for 6 months treatment significantly increased the sperm concentration and delayed the decreased motility with time. None of signs of sperm anomalies and testicular damages were observed [36]. Subchronic treatment of B. superba tuberous powder suspension at high doses $(200 \mathrm{mg} / \mathrm{kg})$ in male rats exhibited adverse effects to blood chemistry, haematology, and blood testosterone level. Powdered crude drug at the doses of 2 , 25,250 , and $1250 \mathrm{mg} / \mathrm{kg}$ body weight was administered for 8 weeks; there was an increased testis weight and sperm counts in rat. Hematology as well as the liver and kidney function of all treated groups showed no difference from the control [37]. A dose-dependent decrease of only blood testosterone, but not LH, was significantly different from the control in the rats treated with high doses of plant powder. This present study suggests that testosterone disruption is significant, at least after 90 days of consumption of high doses of $B$. superba powder [38]. The ethanol extract of B. superba is effective in enhancing penile erection. The ethanol extract increased intracavernous pressure (ICP) in vivo. It also significantly 
TABLE 2: A list of few of the various popular herbs with ehtnopharmacological backing for being used as aphrodisiac. The table also describes the possible mechanism of action of one or more constitutent isolated from them.

\begin{tabular}{|c|c|c|c|c|c|}
\hline $\begin{array}{l}\text { Serial } \\
\text { number }\end{array}$ & Biological source & Part used & Extract used & Mechanism of action & References \\
\hline 1 & $\begin{array}{l}\text { Aframomum Melegueta } \\
\text { Roscoe (Zingiberaceae) }\end{array}$ & Fruits & Aqueous extracts & $\begin{array}{l}\text { Improvement of sexual } \\
\text { behaviour }\end{array}$ & {$[114]$} \\
\hline 2 & $\begin{array}{l}\text { Asparagus racemosus Willd. } \\
\text { (Liliaceae) }\end{array}$ & Roots & Aqueous extracts & $\begin{array}{l}\text { Improvement of sexual } \\
\text { behaviour }\end{array}$ & {$[115]$} \\
\hline 3 & $\begin{array}{l}\text { Allium tuberosum Rottl. ex Spreng } \\
\text { (Liliaceae) }\end{array}$ & Seeds & Butanolic extract & $\begin{array}{l}\text { Improvement of sexual } \\
\text { behaviour }\end{array}$ & {$[116]$} \\
\hline 4 & $\begin{array}{l}\text { Alpinia calcarata Roscoe } \\
\text { (Zingiberaceae) }\end{array}$ & Rhizomes & Hot Aqueous extract & $\begin{array}{l}\text { Increase of sexual behaviour } \\
\text { And testosterone }\end{array}$ & {$[117]$} \\
\hline 5 & $\begin{array}{l}\text { Argeria nervosa syn. A. speciosa } \\
\text { Sweet } \\
\text { (Convolvulaceae) }\end{array}$ & $\begin{array}{l}\text { Fresh leaves, } \\
\text { roots and } \\
\text { flowers }\end{array}$ & $\begin{array}{l}\text { Aqueous, ethanol, } \\
\text { hexane extract }\end{array}$ & $\begin{array}{l}\text { Improvement of sexual } \\
\text { behaviour }\end{array}$ & {$[118]$} \\
\hline 6 & $\begin{array}{l}\text { Aspidosperma ulei Markgr. } \\
\text { (Apocyanaceae) }\end{array}$ & Root & Alkaloidal rich fraction & Increase penile erection & [119] \\
\hline 7 & $\begin{array}{l}\text { Asteracantha longifolia Nees (Syn) } \\
\text { (Acanthaceae) }\end{array}$ & Seeds & Ethanolic extract & $\begin{array}{l}\text { Improvement of sexual } \\
\text { behaviour }\end{array}$ & {$[120]$} \\
\hline 8 & $\begin{array}{l}\text { Anacyclus pyrethrum DC } \\
\text { (Compositae) }\end{array}$ & Root & $\begin{array}{l}\text { Aqueous extract, } \\
\text { Ethanolic extract }\end{array}$ & $\begin{array}{l}\text { Improvement of sexual } \\
\text { behaviour }\end{array}$ & {$[121-123]$} \\
\hline 9 & $\begin{array}{l}\text { Anacardium occidentale } \mathrm{L} \text {. } \\
\text { (Anacardiaceae) }\end{array}$ & Leaf & $\begin{array}{l}\text { Hexane } \\
\text { extract }\end{array}$ & Increase fertility & {$[124]$} \\
\hline 10 & $\begin{array}{l}\text { Bulbine natalensis Baker } \\
\text { (Asphodelaceae) }\end{array}$ & Stem & Aqueous extract & Increase hormone level & {$[125]$} \\
\hline 11 & $\begin{array}{l}\text { Butea Frondosa Koen. ex Roxb. } \\
\text { (Leguminosae) }\end{array}$ & Bark & Aqueous extract & $\begin{array}{l}\text { Improvement of sexual } \\
\text { behaviour }\end{array}$ & [126] \\
\hline 12 & Butea superba Roxb (Leguminosae) & Tuber & Ethanolic extracts & Increase penile erection & [39] \\
\hline 13 & $\begin{array}{l}\text { Bryonia lacinosa Linn } \\
\text { (Cucurbitaceae) }\end{array}$ & Seeds & Ethanolic extracts & $\begin{array}{l}\text { Improvement of sexual } \\
\text { behaviour }\end{array}$ & {$[127]$} \\
\hline 14 & $\begin{array}{l}\text { Basella alba L. } \\
\text { (Basellaceae) }\end{array}$ & Leaves & $\begin{array}{l}\text { Terpenoid } \\
\text { or steroid compounds }\end{array}$ & $\begin{array}{l}\text { Increased the blood testosterone } \\
\text { concentrations }\end{array}$ & {$[128]$} \\
\hline 15 & $\begin{array}{l}\text { Boesenbergia rotunda (L.) Mansf. } \\
\text { (Zingiberaceae) }\end{array}$ & Rhizome & Ethanolic extract & $\begin{array}{l}\text { Improvement of sexual } \\
\text { behaviour }\end{array}$ & [129] \\
\hline 16 & $\begin{array}{l}\text { Casimiroa edulis La Llave } \\
\text { (Rutaceae) }\end{array}$ & Seed & Aqueous extract & Improve sexual activity & {$[130]$} \\
\hline 17 & $\begin{array}{l}\text { Camellia sinensis (L.) O. Kuntze } \\
\text { (Theaceae), }\end{array}$ & $\begin{array}{l}\text { Leaves and } \\
\text { buds }\end{array}$ & Aqueous extract & $\begin{array}{l}\text { Increased the blood testosterone } \\
\text { concentrations }\end{array}$ & {$[131]$} \\
\hline 18 & $\begin{array}{l}\text { Catha edulis Forsk } \\
\text { (Celestreceae) }\end{array}$ & $\begin{array}{l}\text { Shoots and } \\
\text { Small } \\
\text { branches }\end{array}$ & $\begin{array}{l}\text { Chloroform : diethyether } \\
\quad \text { extract }(1: 3)\end{array}$ & $\begin{array}{l}\text { Improvement of sexual } \\
\text { behaviour }\end{array}$ & {$[132]$} \\
\hline 19 & $\begin{array}{l}\text { Caesalpinia benthamiana L. } \\
\text { (Caesalpiniaceae) }\end{array}$ & Roots & Aqueous extract & Improve sexual activity & {$[133]$} \\
\hline 20 & $\begin{array}{l}\text { Chlorophytum borivilianum Sant. } \\
\text { (Liliaceae) }\end{array}$ & Roots & Ethanolic extract & Improve sexual activity & {$[51]$} \\
\hline 21 & $\begin{array}{l}\text { Cocculus hirsutus } \\
\text { Linn (Menispermaceae) }\end{array}$ & Aerial part & Methanolic extract & Spermatogenic & [134] \\
\hline 22 & $\begin{array}{l}\text { Curculigo orchioides Gaertn } \\
\text { (Amaryllidaceae) }\end{array}$ & Rhizome & Ethanolic extract & Improve sexual activity & {$[42,135]$} \\
\hline 23 & Crocus sativus L. (Iridaceae) & Stigma & Aqueous extract & Improve sexual activity & [136] \\
\hline 24 & $\begin{array}{l}\text { Cynomorium coccineum L. } \\
\text { (Cynomoraceae) }\end{array}$ & Roots & $\begin{array}{l}\text { Water } \\
\text { Extract }\end{array}$ & $\begin{array}{l}\text { Increased spermatogenesis and } \\
\text { increase sperm count }\end{array}$ & {$[48]$} \\
\hline 25 & $\begin{array}{l}\text { Diodia scandeus } \\
\text { SW. (Rubiaceae) }\end{array}$ & Herbs & Ethanolic extract & $\begin{array}{l}\text { Potentiates the action of } \mathrm{ACh} \\
\text { and adrenaline }\end{array}$ & {$[137]$} \\
\hline 26 & $\begin{array}{l}\text { Dracaena arborea (Wild) Link } \\
\text { (Dracaenaceae) }\end{array}$ & Root & Aqueous and Ethanolic & Improve sexual activity & [138] \\
\hline 27 & $\begin{array}{l}\text { Dactylorhiza hatagirea (D.Don) Soo } \\
\text { (Orchidacea) }\end{array}$ & Tubers & Aqueous extract & Improve sexual activity & [139] \\
\hline
\end{tabular}


TABLe 2: Continued.

\begin{tabular}{|c|c|c|c|c|c|}
\hline $\begin{array}{l}\text { Serial } \\
\text { number }\end{array}$ & Biological source & Part used & Extract used & Mechanism of action & References \\
\hline 28 & $\begin{array}{l}\text { Eurycoma longifolia Jack. } \\
\text { (Simaroubaceae) }\end{array}$ & Roots & Methanolic extracts & Improve sexual activity & {$[67]$} \\
\hline 29 & Eulophia nuda Lind. (Orchidaceae) & Tubers & Powder & Improve sexual activity & {$[140]$} \\
\hline 30 & $\begin{array}{l}\text { Epimedium koreanum Nakai } \\
\text { (Berberidaceae) }\end{array}$ & Herbs & Aqueous and Ethanolic & Improve sexual activity & {$[56]$} \\
\hline 31 & $\begin{array}{l}\text { Epimedium brevicornum } \\
\text { Maxim (Berberidaceae) }\end{array}$ & Root & Aqueous Extract & Increase Nitric oxide release & {$[141]$} \\
\hline 32 & $\begin{array}{l}\text { Fadogia agrestis Schweinf. Ex Hiern } \\
\text { (Rubiaceae) }\end{array}$ & Stem & $\begin{array}{l}\text { Alkaloids and } \\
\text { Saponins }\end{array}$ & $\begin{array}{l}\text { Increased the blood testosterone } \\
\text { concentrations }\end{array}$ & {$[142]$} \\
\hline 33 & $\begin{array}{l}\text { Ferula hermonis Boiss } \\
\text { (Umbelliferae) }\end{array}$ & Roots & Acetonic extract & Stimulated sexual motivation & [143] \\
\hline 34 & $\begin{array}{l}\text { Garcinia cambogia Desr. } \\
\text { (Clusiaceae) }\end{array}$ & Seeds & $\begin{array}{l}\text { Biflavonoid and } \\
\text { xanthone }\end{array}$ & Increase sperm count & {$[144]$} \\
\hline 35 & $\begin{array}{l}\text { Hibiscus sabdariffa L. } \\
\text { (Malvaceae) }\end{array}$ & Flowers & Anthocyanins & Increase Sperm count & {$[145]$} \\
\hline 36 & $\begin{array}{l}\text { Hibiscus macranthus } \\
\text { Hochst. Ex A.Rich. } \\
\text { (Malvaceae) }\end{array}$ & Leaves & $\begin{array}{l}\text { Terpenoid } \\
\text { or steroid compounds }\end{array}$ & $\begin{array}{l}\text { Increased the blood testosterone } \\
\text { concentrations }\end{array}$ & [128] \\
\hline 37 & $\begin{array}{l}\text { Kaempferia parviflora Wall. Ex. } \\
\text { Baker } \\
\text { (Zingiberaceae) }\end{array}$ & Rhizomes & Alcohol extract & $\begin{array}{l}\text { Increase in blood flow to the } \\
\text { testis, Sexual behaviour }\end{array}$ & {$[146,147]$} \\
\hline 38 & $\begin{array}{l}\text { Lepidium meyenii } \\
\text { Walpers (Brassicaceae) }\end{array}$ & Root & Aqueous extract & Spermatogenesis & {$[83]$} \\
\hline 39 & Lophira laceolata (Ohanacea) & Stem Bark & Aqueous extract & Improve sexual behaviour & {$[148]$} \\
\hline 40 & $\begin{array}{l}\text { Lithospermum arvense } \mathrm{L} \text {. } \\
\text { (Boraginaceae) }\end{array}$ & Seed & Aqueous extract & Androgenic & {$[149]$} \\
\hline 41 & $\begin{array}{l}\text { Massularia acuminata (G. Don) } \\
\text { Bullock ex Hoyl. (Rubiaceae) }\end{array}$ & Stem & Aqueous extract & Stimulate male sexual maturation & {$[150]$} \\
\hline 42 & $\begin{array}{l}\text { Mondia whitei (Hook f.) Skeels. } \\
\text { (Periplocaceae) }\end{array}$ & Roots & Aqueous extract & $\begin{array}{l}\text { Increase in the testicular weight } \\
\text { and serum and testicular } \\
\text { testosterone }\end{array}$ & {$[151,152]$} \\
\hline 43 & $\begin{array}{l}\text { Mucuna pruriens Baker } \\
\text { (Fabaceae) }\end{array}$ & Seed & Chloroform extract & Spermatogenic & {$[84]$} \\
\hline 44 & $\begin{array}{l}\text { Microdesmis keayana J.Léonard. } \\
\text { (Pandaceae) }\end{array}$ & Roots & $\begin{array}{l}\text { Keayanidine } \mathrm{B} \text { and } \\
\text { keayanine }\end{array}$ & Stimulate NO production & {$[153]$} \\
\hline 45 & $\begin{array}{l}\text { Montanoa tomentosa Cerv } \\
\text { (Asteraceae) }\end{array}$ & $\begin{array}{l}\text { Leaves and } \\
\text { flowers }\end{array}$ & Aqueous extract & Improve sexual behaviour & {$[154]$} \\
\hline 46 & $\begin{array}{l}\text { Myristica fragrans } \\
\text { Houtt. (Myristicaceae) }\end{array}$ & Nutmeg & $50 \%$ ethanolic extracts & Improve sexual behaviour & {$[155,156]$} \\
\hline 47 & Orchis latifolia Linn (Orchidaceae) & Roots & $\begin{array}{l}\text { Aqueous extract, } \\
\text { Powder }\end{array}$ & Improve sexual behaviour & {$[140,157]$} \\
\hline 48 & $\begin{array}{l}\text { Panax ginseng } \\
\text { C. A. Mayer (Araliaceae) }\end{array}$ & Roots & $\begin{array}{l}\text { Ginsenosides, } \\
\text { saponin glycosides }\end{array}$ & $\begin{array}{l}\text { Increase Nitric oxide central } \\
\text { nervous system action }\end{array}$ & {$[158]$} \\
\hline 49 & $\begin{array}{l}\text { Panax quinquefolium } \\
\text { L. (Araliaceae) }\end{array}$ & Roots & Powdered & $\begin{array}{l}\text { Facilitate male copulatory } \\
\text { behavior }\end{array}$ & {$[159]$} \\
\hline 50 & $\begin{array}{l}\text { Pausinystalia yohimbe } \\
\text { Pierre ex Beille (Rubiaceae) }\end{array}$ & Bark & Yohimbine & $\begin{array}{l}\text { Improve sexual behaviour } \\
\text { And penile erection }\end{array}$ & {$[160]$} \\
\hline 51 & $\begin{array}{l}\text { Peganum harmala L. } \\
\text { (Zygophyllaceae) }\end{array}$ & Seed & Powdered & $\begin{array}{l}\text { Improve semen quality, } \\
\text { Spermatogenesis and organ } \\
\text { weight }\end{array}$ & {$[161]$} \\
\hline 52 & $\begin{array}{l}\text { Pentadiplandra brazzeana } \\
\text { Baill. (Capparidaceae) }\end{array}$ & Root & Aqueous extract & $\begin{array}{l}\text { Serum and testicular testosterone } \\
\text { levels testicular cholesterol, the } \\
\text { seminal vesicular fructose }\end{array}$ & {$[162]$} \\
\hline 53 & $\begin{array}{l}\text { Pfaffia paniculata (Martius) Kuntze } \\
\text { (Amaranthaceae) }\end{array}$ & Root & Aqueous extract & Increase serum testosterone & {$[163]$} \\
\hline
\end{tabular}


TABLe 2: Continued.

\begin{tabular}{|c|c|c|c|c|c|}
\hline $\begin{array}{l}\text { Serial } \\
\text { number }\end{array}$ & Biological source & Part used & Extract used & Mechanism of action & References \\
\hline 54 & $\begin{array}{l}\text { Piper Gnineense Schumach. } \\
\text { \& Thonn. (Piperaceae) }\end{array}$ & Fruits & Aqueous extract & $\begin{array}{l}\text { Improvement of sexual } \\
\text { behaviour }\end{array}$ & {$[114]$} \\
\hline 55 & Psidium guajava Linn (Myrtaceae) & Leaves & Ethanol extract & Increase sperm count & {$[164]$} \\
\hline 56 & $\begin{array}{l}\text { Psoralea corylifolia } \mathrm{L} . \\
\text { (Fabaceae) }\end{array}$ & Fruits & Aqueous extract & $\begin{array}{l}\text { Increased sperm counts, induces } \\
\text { spermatogenesis }\end{array}$ & {$[165]$} \\
\hline 57 & $\begin{array}{l}\text { Passiflora incarnate Linn } \\
\text { (Passifloraceae) }\end{array}$ & Leaves & Methanolic extract & Increase sexual behaviour & {$[166]$} \\
\hline 58 & Rubus coreanus Miq (Rosaceae) & Fruit & Powder & Enhancing spermatogenesis & {$[167]$} \\
\hline 59 & $\begin{array}{l}\text { Rhoicissus tridentata L.f. (Wild and } \\
\text { R.B. Drumm) } \\
\text { (Vitaceae) }\end{array}$ & Root bark & $\begin{array}{l}\text { Chloroform and } \\
\text { Ethanolic extract }\end{array}$ & $\begin{array}{l}\text { Relaxed the corpus cavernosal } \\
\text { smooth muscle }\end{array}$ & {$[168]$} \\
\hline 60 & $\begin{array}{l}\text { Syzygium aromaticum (L.) Merr. \& } \\
\text { Perry. }\end{array}$ & Flower bud & $50 \%$ ethanolic extract & Increase sexual behaviour & {$[169]$} \\
\hline 61 & $\begin{array}{l}\text { Senecio cardiophyllus Hemsl } \\
\text { (Asteraceae) }\end{array}$ & Root & Aqueous extract & Increase the ejaculatory capacity & {$[170]$} \\
\hline 62 & $\begin{array}{l}\text { Salvia haematodes L. } \\
\text { (Lamiaceae) }\end{array}$ & Roots & Ethanolic extract & Increase sexual behaviour & {$[171]$} \\
\hline 63 & $\begin{array}{l}\text { Securidaca longepedunculata } \\
\text { (Fresen) (Polygalaceae), }\end{array}$ & Root bark & $\begin{array}{l}\text { Chloroform and } \\
\text { Ethanolic extract }\end{array}$ & $\begin{array}{l}\text { Relaxed the corpus cavernosal } \\
\text { smooth muscle }\end{array}$ & {$[168]$} \\
\hline 64 & $\begin{array}{l}\text { Terminalia catappa Linn. } \\
\text { (Combretaceae) }\end{array}$ & Seeds & Seed suspension & Increase sexual behaviour & {$[172]$} \\
\hline 65 & $\begin{array}{l}\text { Tribulus terrestris } \mathrm{L} \text {. } \\
\text { (Zygophyllaceae) }\end{array}$ & Fruits & Protodioscin & Androgen increasing property & {$[101]$} \\
\hline 66 & $\begin{array}{l}\text { Tribulus alatus } \\
\text { Delile (Zygophyllaceae) }\end{array}$ & $\begin{array}{l}\text { Aerial parts } \\
\text { and fruits }\end{array}$ & $70 \%$ alcoholic extract & Increase serum testosterone & {$[173]$} \\
\hline 67 & $\begin{array}{l}\text { Trichopus Zeylanicus } \\
\text { Gaertn (Trichopodaceae) }\end{array}$ & Leaf & Ethanolic extract & Stimulate sexual behaviour & {$[174]$} \\
\hline 68 & $\begin{array}{l}\text { Tricholepis glaberrima. DC } \\
\text { (Compositae) }\end{array}$ & Aerial parts & Methanol extract & Stimulate sexual behaviour & {$[175]$} \\
\hline 69 & $\begin{array}{l}\text { Turnera diffusa Willd } \\
\text { (Turneraceae) }\end{array}$ & Leaf & $\begin{array}{l}30 \% \text { ethanol in water } \\
(\mathrm{v}: \mathrm{v})\end{array}$ & Stimulate sexual behaviour & {$[176]$} \\
\hline 70 & $\begin{array}{l}\text { Vanda tessellate (Roxb.) } \\
\text { Hook. Ex Don } \\
\text { (Orchidaceaae) }\end{array}$ & $\begin{array}{l}\text { Root, } \\
\text { flower }\end{array}$ & Aqueous and ethanolic & Stimulate sexual behaviour & {$[177]$} \\
\hline 71 & $\begin{array}{l}\text { Wrightia natalensis (Stapf) } \\
\text { (Apocynaceae) }\end{array}$ & Root bark & $\begin{array}{l}\text { Chloroform and } \\
\text { Ethanolic extract }\end{array}$ & $\begin{array}{l}\text { Relaxed the corpus cavernosal } \\
\text { smooth muscle }\end{array}$ & {$[168]$} \\
\hline 72 & $\begin{array}{l}\text { Withania somnifera (L.) Dunal. } \\
\text { (Solanaceae) }\end{array}$ & Root & Aqueous extract & Spermatogenesis & {$[49]$} \\
\hline 73 & $\begin{array}{l}\text { Zingiber officinale } \\
\text { Roscoe (Zingiberaceae) }\end{array}$ & Roots & Aqueous extract & $\begin{array}{l}\text { Increase of both testis weight and } \\
\text { serum testosterone } \\
\text { levels }\end{array}$ & {$[162]$} \\
\hline 74 & $\begin{array}{l}\text { Pueraria tuberosa DC } \\
\text { (Fabaceae) }\end{array}$ & tubers & Ethanolic extract & Stimulate sexual behavior & {$[178]$} \\
\hline 75 & $\begin{array}{l}\text { Spilanthes acmella } \\
\text { Murr. (Asteraceae) }\end{array}$ & flowers & Ethanolic extract & Stimulate sexual behavior & {$[179]$} \\
\hline 76 & $\begin{array}{l}\text { Pedalium murex Linn. } \\
\text { (Pedaliaceae) }\end{array}$ & fruits & Ethanolic extract & Stimulate sexual behavior & {$[180]$} \\
\hline 77 & $\begin{array}{l}\text { Dracaena arboreaI (Willd.) } \\
\text { (Asparagaceae) }\end{array}$ & Roots & $\begin{array}{l}\text { Aqueous and ethanolic } \\
\text { extracts }\end{array}$ & $\begin{array}{l}\text { inhibit the activity of the } \\
\text { bulbospongiosus muscles }\end{array}$ & {$[181]$} \\
\hline 78 & $\begin{array}{l}\text { Allanblackia floribunda } \\
\text { Oliv. (Guttiferae) }\end{array}$ & Stem bark & $\begin{array}{l}\text { Aqueous and ethanolic } \\
\text { extracts }\end{array}$ & $\begin{array}{l}\text { inhibit the activity of the } \\
\text { bulbospongiosus muscles }\end{array}$ & {$[182]$} \\
\hline 79 & Corchorus depressus Linn (Tiliaceae) & Whole plant & Chlorofrm fraction & Stimulate sexual behavior & {$[183]$} \\
\hline 80 & Arctium lappa L (Asteraceae) & Root & Aqueous extract & Stimulate sexual behavior & {$[184]$} \\
\hline 81 & Musa paradisiacal L (Musaceae) & Root & Aqueous extract & Increase testosterone level & {$[185]$} \\
\hline
\end{tabular}


TABLe 2: Continued.

\begin{tabular}{llccc}
\hline $\begin{array}{l}\text { Serial } \\
\text { number }\end{array}$ & Biological source & Part used & Extract used & Mechanism of action \\
\hline 82 & $\begin{array}{l}\text { Lecaniodiscus cupanioides } \\
\text { Planch. (Sapindaceae) }\end{array}$ & Root & Aqueous extract & Increase testosterone level \\
\hline
\end{tabular}

TABLE 3: A tabular overview of some of the active consitutent with scientific findings and source description.

\begin{tabular}{|c|c|c|c|}
\hline Serial number & Plant name & Active constituent & References \\
\hline 1 & Andrographis paniculata Wall.ex Nees (Acanthaceae) & Andrographolide & [187] \\
\hline 2 & Aspidosperma ulei Markgr (Apocyanaceae) & Alkaloidal fraction & [119] \\
\hline 3 & Catha edulis (Vahl) Forssk. ex Endl. (Celastraceae) & Cathinone & [188] \\
\hline 4 & Crocus sativus L. (Iridaceae) & Crocetin, Crocin & [136] \\
\hline 5 & Epimedium sagittatum (Siebold \& Zucc.) Maxim. (Berberidaceae) & Icariin & {$[58]$} \\
\hline 6 & Ferula hermonis L. (Umbelliferae) & Ferutinin & {$[189,190]$} \\
\hline 7 & Panax ginseng L. (Araliaceae) & Ginsenosides & {$[158,191]$} \\
\hline 8 & Lepedium meyeni Walp (Oleacaceae) & Meiconoides & {$[71]$} \\
\hline 9 & Lycium barbaru L. (Solanaceae) & Polysaccharides & [192] \\
\hline 10 & Mucuna macrocarpa Wall. (Leguminosae) & Quercetin & [193] \\
\hline 11 & Mucuna pruriens Baker. (Leguminosae) & Total Alkaloids & {$[84]$} \\
\hline 12 & Microdesmis keayana J. Léonard (Pandaceae) & Keayanidine B and keayanine & [153] \\
\hline 13 & Palisota Hirsuta (Thunb.) K. Schum. (Commelinaceae) & Total Flavonoids & {$[194]$} \\
\hline 14 & Pausinystalia yohimbe Pierre ex Beille (Rubiaceae) & Yohimbine & {$[195,196]$} \\
\hline 15 & Securidaca longepedunculata Fres (Polygalaceae) & Novel xanthones, & [197] \\
\hline 16 & Satureja khuzestanica Jamzad (Lamiaceae) & Essential oil; & [198] \\
\hline 17 & Tribulus terrestris L. (Zygophyllaceae) & Saponins, Furostenol Glycoside & {$[100,199]$} \\
\hline 18 & Turnera diffusa Wild (Turneraceae) & Flavonoids & [200] \\
\hline 19 & Zingiber officinale Roscoe (Zingiberaceae) & Zingerone, Gingerdiol & {$[145]$} \\
\hline 20 & Vanda tessellata (Roxb.) ex Don (Orchidaceae) & 2,7,7-tri methyl bicyclo [2.2.1] heptane & {$[201]$} \\
\hline 21 & Smallanthus sonchifolius Yacon (Asteraceae) & ferulic acid, chlorogenic acid & {$[202]$} \\
\hline
\end{tabular}

enhanced the effects of cGMP and isobutylmethylxanthine. This suggests that $B$. superba may act through cAMP/cGMP pathways [39].

Clinical Studies. The plant powder showed potential activity in a human clinical trial for treatment of erectile dysfunction in males [40].

10.2. Curculigo orchioides. Curculigo orchioides Gaertn (Amaryllidaceae), also known as Kali Musli or Syah (black) Musli, is considered as aphrodisiac and Rasayan or rejuvenator [41]. The ethanolic extract of rhizome improved sexual behaviour in male rats. The sexual performance as assessed by determining parameters such as penile erection, mating performance, and sexual and orientation behavior was significantly improved. Moreover a pronounced anabolic and spermatogenic effect was evidenced by weight gains of reproductive organs. The treatment also markedly affected sexual behavior of animals as reflected in reduction of mount latency, an increase in mount frequency and enhanced attractability towards female. Penile erection index was also incremented in treated group $[42,43]$. The lyophilized aqueous extracts of Curculigo orchioides significantly improved the pendiculatory activity in male rats after 14 days of treatment. Similarly, the extract could also preserve the in vitro sperm count when compared to control group after $30 \mathrm{~min}$. of incubation [44]. The aqueous extract of the plant showed prominent activity at a dose level of $200 \mathrm{mg} / \mathrm{kg}$. In general, a pronounced anabolic effect in treated animals was evidenced by weight gains in the body and reproductive organs. There was a significant variation in the sexual behavior of animals as reflected by reduction of mount latency, ejaculation latency, postejaculatory latency, intromission latency, and an increase of mount frequency. Penile erection was also considerably enhanced. Reduced hesitation time (an indicator of attraction towards female in treated rats) also indicated an improvement in sexual behavior of extract treated animals [45]. In case of physically induced sexual dysfunction, that is, heat induced damaged to the testicular function, the plant was useful in ameliorating the reduced spermatogenesis and the treated animals could effectively overcome the heat shock protein; this exemplified the role of $C$. orchioides in overcoming physically induced sexual dysfunction due to testicular damage [46].

10.3. Cynomorium coccineum. Cynomorium coccineum Linn. (Cynomoraceae) is known as Som-El-Ferakh in Saudi Arabia, which is a black leafless parasitic plant devoid of chlorophyll. The natives in Qatar use it (mainly with honey) as a tonic and aphrodisiac [47]. Aqueous extract of Cynomorium coccineum 
induced significant increase in the sperm count, improved the percentage of live sperm and their motility, and decreased the number of abnormal sperm. Testicular histology showed increased spermatogenesis and seminiferous tubules full of sperm in the treated group compared to the untreated controls [48]. Aqueous extract of the plant elicited notable spermatogenesis in immature rats. Serum testosterone and FSH levels were lower in animals treated with extracts than controls, whereas interstial cell stimulating hormone levels was higher in treated animals [49].

10.4. Chlorophytum borivilianum. Safed Musli (Chlorophytum borivilianum) belongs to the family Liliaceae with folkloric claims as aphrodisiac and sexual stimulant [50]. Ethanolic extract of roots as well as sapogenins isolated from the roots were studied for effect on sexual behavior and spermatogenesis in albino rats. Treatment had pronounced anabolic and spermatogenic effect in treated animals, evidenced by weight gains of body and reproductive organs. Administration of extracts markedly affected sexual behavior of animals reflected in reduction of mount ejaculation, postejaculatory, and intromission latency. An increase in mount frequency and attractability towards female was observed [51]. The aqueous extract of dried roots of Chlorophytum borivilianum enhances the sexual arousal, vigor, and libido in Wistar rats. The extract increases sperm count significantly $[44,52]$.

In case of streptozotocin and alloxan induced hyperglycemia, the aqueous extract from the plant resulted in amelioration of sexual dysfunction, resulted in improved sexual performance compared to diabetic control rats. The study thus provided evidence that herbal drugs may act on sexual dysfunction in normal as well as diabetic animals $[53,54]$.

10.5. Epimedium koreanum. The traditional Chinese medicinal herb, Epimedium L. (Berberidaceae), is a popular botanical supplement used as a health tonic. Most important Epimedium species used for medicinal purposes are E. koreanum Nakai, E. pubescens Maxim., E. brevicornum Maxim, E. sagittatum (Sieb. Et Zucc) Maxim, and E. wushanense T.S. Ying [55]. Hydroalcholic extract of the plants are reputed to produce aphrodisiac effects and are commonly used in Chinese herbal medicine to enhance erectile function [56]. It is thought that icariin is likely to be the primary active component of Epimedium extracts. Icariin is a flavonol, a type of flavonoid. It is the prenylacetylation of kaempferide 3,7-O-diglycoside, icariin on erectile dysfunction and established its dose-dependent selective inhibitory effect on phosphodiesterase-5 (PDE5). Oral treatment with icariin (>98.6\% purity) for 4 weeks potentially improves erectile function. This effect is correlated with an increase in the percentage of smooth muscle and the expression of certain NO synthase isoforms in the corpus cavernosum of castrated rats. These results suggest that icariin may have a therapeutic effect on erectile dysfunction [57]. Icariin was inhibitory to all three PDE5 isoforms and with similar IC50 values, which were approximately three times greater than those for zaprinast. Icariin was able to enhance the levels of cyclic guanosine monophosphate in sodium nitroprusside-treated cavernous smooth muscle cells [58-60] and to enhance the production of bioactive nitric oxide [61] as well as mimicking the effects of testosterone [62].

10.6. Eurycoma longifolia. Eurycoma longifolia Jack (Simaroubaceace), known locally as Tongkat Ali, is commonly found in lowland forests. It is very commonly used by ethnic groups for numerous reasons and is one of the major export components from Malaysia [63]. E. longifolia increases sexual motivation in sexually naive male rats. An electric grid was used as an obstruction in the electrical copulation cage in order to determine how much an aversive stimulus the sexually naive male rat for both the treated with $E$. longifolia and control groups were willing to overcome to reach the estrous receptive female in the goal cage. The intensity of the grid current was maintained at $0.12 \mathrm{~mA}$ and this was the intensity in which the male rats in the control group failed to crossover to reach the goal cage. Results showed that E. longifolia Jack continued to enhance and also maintain a high level of both the total number of successful crossovers, mountings, intromissions, and ejaculations during the 9-12th week observation period [64]. Ethanol extract treatment for 10 days increased the sexual performance of the treated male rats by extending the duration of coitus and decreasing the refractory period between the different series of copulation [65]. Administration of $800 \mathrm{mg} / \mathrm{kg}$ of butanol, methanol, water, and chloroform fractions of E. longifolia significantly increased the leavator ani muscle when compared with the control (untreated) in the uncastrated intact male rats and when compared to control (untreated) in the testosteronestimulated castrated intact male rats [66]. E. longifolia continued to enhance and also maintain a high level of both the total number of successful crossovers, mountings, intromissions, and ejaculations during the 9-12th week observation period. Butanol, methanol, water, and chloroform extracts of the roots of E. longifolia produced a dose-dependent, recurrent, and significant increase in the episodes of penile reflexes as evidenced by increases in quick flips, long flips, and erections of the treated male rats during the 30 -min observation period [67]. E. longifolia $(0.5 \mathrm{~g} / \mathrm{kg})$ for three-week increase in the percentage of the male rats responding to the right choice, more than $50 \%$ of the male rats scored "right choice" after 3 weeks posttreatment and the effect became more prominent after 8 weeks posttreatment (only $40-50 \%$ of the control male rats responded to the right choice) using the electrical copulation cage [68]. The middle-aged male rats treated with $800 \mathrm{mg} / \mathrm{kg}$ of E. longifolia increased orientation activities towards the receptive females as evidenced by increase in anogenital investigatory behaviour, licking, and mounting but possessed a lack of interest in the external environment as evidenced by decrease in climbing, raring, and exploration on the caged wall, as well as it also enhanced self-orientation as evidenced by increased grooming of their own genitals and also showed restricted confinement, with targeted orientation and movement toward female as compared with the controls; it also enhanced the sexual qualities of the middle-aged male rats by decreasing their hesitation time as compared to controls with various fractions of $E$. longifolia $[69,70]$. 
10.7. Lepidium meyenii. Lepidum meyenii Walp (Brassicaceae) known as Maca is the edible root traditionally employed for its purported aphrodisiac and fertilityenhancing properties. Subacute oral administration of hexanic, methanolic, and chloroform extracts of Maca (Lepidium meyenii) root significantly decreased intromission latency and intercopulatory interval and increased intromission frequency and copulatory efficacy as compared to controls. Hexanic and methanolic extracts were able to increase mount frequency, while only hexanic fraction significantly improved mount latency. Globally, only the hexanic fraction significantly improved the majority of the sexual parameters measured. Subacute oral administration of hexanic Maca extract improved sexual performance parameters in sexually inexperienced male rats most effectively [71]. Oral administration of lipidic extract from Lepidium meyenii enhanced the sexual function of the mice and rats, as evidenced by an increase in the number of complete intromissions and the number of sperm-positive females in normal mice, and a decrease in the latent period of erection in male rats with erectile dysfunction [72]. Improvement of larginine-nitric oxide activity has also been attributed to Maca. The acute and daily administration of Maca in sexually experienced male rats produced a small change in ejaculation latency and postejaculatory interval and these changes disappeared with chronic treatment. Chronic administration of Maca did not increase anxiety and had some effect on locomotor activity [73]. Black maca appeared to have more beneficial effects on sperm counts and epididymal sperm motility after 42 days of treatment [74]. Maca has also shown effectiveness as a lead treatment for the dysfunction arising due to metalic lead exposure. Maca protects spermatogenesis by increasing lengths of stages VIII and IX-XI and daily sperm count that result in an increase in epididymal sperm number [75]. Oral treatment with ethyl acetate fraction of the hydroalcoholic extract of Black Maca for 7 days had the most beneficial effect on epididymal sperm count and daily sperm count compared with other fractions [76].

Clinical Studies. Maca enhanced fertility in both men and women $[77,78]$. Improvement of sexual desire is not related to changes in pituitary or gonadal hormones $[79,80]$. Maca does not activate androgen receptors and may actually block androgen receptors $[81,82]$. Maca aqueous extract can be considered safe in doses up to $5 \mathrm{~g}$ extract $/ \mathrm{kg}$, corresponding to some $11 \mathrm{~g}$ dry hypocotyls $/ \mathrm{kg}$. The effect on reproductive physiology may be observed at $0.10 \mathrm{~g}$ extract $/ \mathrm{kg}$ of Maca extract that represents $15.4 \mathrm{~g}$ of dry hypocotyls for an individual of $70 \mathrm{~kg}$ [83].

10.8. Mucuna pruriens. Mucuna pruriens Linn. Family Leguminosae is a popular Indian medicinal plant, which has long been used in traditional Ayurvedic Indian medicine. The total alkaloids from the seeds of $M$. pruriens were found to increase spermatogenesis and weight of the testes, seminal vesicles, and prostate in the albino rat [84]. M. pruriens stimulated sexual function in normal male rats which was observed by increase in mounting frequency, intromission frequency, and ejaculation latency [85]. M. pruriens seed powder improved significantly various sexual parameters copulatory behavior including mount frequency, mount latency, intromission frequency, and intromission latency of the male albino rats [86]. The ethanolic extracts of $M$. pruriens seed produced a significant and sustained increase in the sexual activity of normal male rats at a particular dose $(200 \mathrm{mg} / \mathrm{kg})$. There is significantly increased mounting frequency, intromission frequency, and ejaculation latency and decreased mounting latency, intromission latency, postejaculatory interval, and interintromission interval [87]. M. pruriens efficiently recovered the spermatogenic loss induced due to ethinyl estradiol administration to rats. The recovery is mediated by reduction in ROS level, restoration of MMP, regulation of apoptosis, and eventual increase in the number of germ cells and regulation of apoptosis. The major constituent LDOPA of $M$. pruriens largely accounts for prospermatogenic properties [88]. Administered of seed extract of $M$. pruriens to diabetic rats showed significant improvement in sexual behavior, libido and potency, sperm parameters, DSP, and hormonal levels as compared to diabetic rat without extract treatment [89].

Clinical Studies. The Treatment with M. pruriens seeds increased sperm concentration and motility in all the infertile study groups in man. After the treatment of extract the seminal plasma of all the infertile groups, the levels of lipids, antioxidant vitamins, and corrected fructose were recovered after a decrease in lipid peroxides after treatment Their was recovered sperm concentration significantly in oligozoospermic patients, but sperm motility was not restored to normal levels in astheno-zoospermic men [90]. M. pruriens significantly improved T, LH, dopamine, adrenaline, and noradrenaline levels and reduced levels of FSH and PRL in infertile men. It also significantly recovered sperm count and motility. $M$. pruriens treatment to infertile men regulates steroidogenesis and improves semen quality [91, 92]. Treatment with $M$. pruriens significantly inhibited lipid peroxidation, elevated spermatogenesis, and improved sperm motility of infertile male and also improved the levels of total lipids, triglycerides, cholesterol, phospholipids, and vitamin A, C, and $\mathrm{E}$ and corrected fructose in seminal plasma of infertile men [93]. M. pruriens significantly ameliorated psychological stress and seminal plasma lipid peroxide levels along with improved sperm count and motility. Treatment also restored the levels of SOD, catalase, GSH, and ascorbic acid in seminal plasma of infertile men. It reactivates the antioxidant defense system of infertile men and also helps in the management of stress and improves semen quality [94].

10.9. Tribulus terrestris. The plant Tribulus terrestris Linn. (Zygophyllaceae) popularly known as puncture vine is a perennial creeping herb with a worldwide distribution. Since ancient times it is regarded as an aphrodisiac in addition to its beneficial claims on various ailments such as urinary infections, inflammations, leucorrhoea, oedema, and ascites [95]. T. terrestris has long been used in the traditional Chinese and Indian systems of medicine for the treatment of various ailments and is popularly claimed to improve sexual functions. Administration of T. terrestris to male lambs 
and rams improves plasma testosterone and spermatogenesis [96]. It also found to increase the levels of testosterone, luteinizing hormone [97], dehydroepiandrosterone, dihydrotestosterone, and dehydroepiandrosterone sulphate $[98,99]$. The corpus cavernosal tissues obtained from New Zealand white rabbits following treatment with $T$. terrestris were tested in vitro with various pharmacological agents and electrical field stimulation and was found to have a proerectile effect [100]. T. terrestris has been found to increase sexual behaviour in rats. Treatment of castrated rats with T. terrestris extract showed increase in prostate weight and intracavernosal pressure. There was an improvement of the sexual behaviour parameters as evidenced by increase in mount frequency and intromission frequency; decrease in mount latency, intromission latency, and penile erection index $[101,102]$. T. terrestris administration in rats increased the NADPH-d positive neurons and androgen receptor immunoreactivity in the PVN region. Androgens are known to increase both androgen receptor and NADPH-d positive neurons either directly or by its conversion to oestrogen. The mechanism for the observed increase in AR and NADPH-d positive neurons in the present study is probably due to the androgen increasing property of T. terrestris [103]. T. terrestris also increased the synthesis of cyclic nucleotides in CCSM cells [101]. T. terrestris extract increased the levels of T, DHT, and DHEAS and that the effect was more pronounced in hypogonadal state. Such increase in androgen levels could be the responsible factor for the age-old claims of PTN as an aphrodisiac and therefore T. terrestris may be useful as an adjunct in mild to moderate cases of ED [104]. The ability of tribulus to increase the release of nitric oxide may account for its claims as an aphrodisiac $[100,102]$.

10.10. Withania somnifera. Ashwagandha (Withania somnifera (L.) Dunal, Family: Solanaceae) is also known as Indian ginseng commonly used in Ayurvedic medicine. It is best regarded as adaptogen, tonic with aphrodisiac properties. Some workers have reported the decrease mating behavior and antifertility effects of $W$. somnifera root on in mice [105]. The root extract induced a marked impairment in libido, sexual performance, sexual vigour, and penile erectile dysfunction [106]. It also showed antifertility activity in male rat [107]. But some scientist shows that W. somnifera has the capability of combating stress-induced infertility. It also protects swimming-induced reproductive endocrine dysfunctions in male rat [108]. Aqueous extract improved spermatogenesis, which may be due to increased interstitial cell stimulating hormone and testosterone-like effects as well as the induction of nitric oxide synthase [109].

Clinical Studies. Ashwagandha root extract administered to the oligospermic patients resulted in a significantly greater improvement in spermatogenic activity and serum hormone levels as compared to the placebo treated [110]. Treatment of infertile men with Withania somnifera inhibited lipid peroxidation and protein carbonyl content and improved sperm count and motility. It also recovered the seminal plasma levels of antioxidant enzymes $W$. somnifera root powder when administered in a dosage of $5 \mathrm{~g}$ /day for
3 months to normozoospermic infertile man resulted in a decrease in stress, improved the level of antioxidants, and improved overall semen and vitamins A, C, and E and corrected fructose. Significantly increased serum T and $\mathrm{LH}$ and reduced levels of FSH and PRL in infertile men were observed [111, 112].

\section{Conclusion}

Various herbs have been used by people of different cultures to treat conditions of male infertility or for treatment of reproductive disorders. They have also been advocated for improving sexual desire as well as sexual performance and erectile dysfunction, vasodilatation, increased testosterone level, brain monoamines, effect on pituitary-gonadal axis, and so forth are suggested mechanism for its action of these herbs [113].

In absence of clinical efficacy and safety data on these herbs, people are skeptical to use them. There is an urgent need to conduct clinical studies to support traditional claims and to work out cellular and molecular mechanism involved. Investigations in validation of the herbs will go a long way in management of infertility. Moreover, the cross talk of various pathways involved must also be taken into account to come up with a molecular pathway to find a lead molecule of herbal origin of the treatment of various forms of sexual dysfunction.

\section{Conflict of Interests}

The authors declare that there is no conflict of interests regarding the publication of this paper.

\section{Acknowledgment}

One of the authors, Nagendra Singh Chauhan, thanks AICTE, New Delhi, for providing National Doctoral Fellowship.

\section{References}

[1] WHO, WHO Manual for Standardized Investigation, Diagnosis and Management of the Infertile Male, Cambridge University Press, Cambridge, UK, 2000.

[2] M. Carpentier, S. Sahpaz, and F. Bailleul, "Plants and erectile dysfunction," Phytothérapie, vol. 3, pp. 66-71, 2004.

[3] G. Singh and T. Mukherjee, "Herbal aphrodisiacs: a review," Indian Drugs, vol. 35, no. 4, pp. 175-182, 1998.

[4] A. J. Afolayan and M. T. Yakubu, "Erectile dysfunction management options in Nigeria," Journal of Sexual Medicine, vol. 6, no. 4, pp. 1090-1102, 2009.

[5] E. Noumi, P. H. A. Zollo, and D. Lontsi, "Aphrodisiac plants used in Cameroon," Fitoterapia, vol. 69, no. 2, pp. 125-134, 1998.

[6] W. Low and H. Tan, "Asian traditional medicine for erectile dysfunction," The Journal of Men's Health and Gender, vol. 4, no. 3, pp. 245-250, 2007.

[7] Anonymous, The Ayurvedic Pharmacoepia of India, Part 1-4, Government of India, Ministry of Health and Family Welfare, Department of Indian System of Medicine \& Homeopathy, New Delhi, India, 1999. 
[8] S. C. Upadhya, Kama Sutra of Vatsyayana, Taraporevala, Bombay, India, 1961.

[9] R. K. Sharma, Agnivesas Caraka Samhita, Vol. III, vol. 94 of Chowkhamba Sanskrit Studies, 1988.

[10] H. S. Puri, Rasayana: Ayurvedic Herbs for Longevity and Rejuvenation, CRC Press, New Delhi, India, 2002.

[11] K. L. Bhishagratna, Sushruta Samhita, vol. 2 of An English Translation Based On Original Sanskrit Texts, Varanasi, India, 2nd edition, 1963.

[12] B. Kapp and M. Cain, "The neural basis of arousal," in The International Encyclopedia of Social and Behavioral Sciences, N. Smelser and P. Baltes, Eds., Elsevier Science, Oxford, UK, 2001.

[13] M. R. Melis and A. Argiolas, "Neuropeptides and central control of sexual behaviour from the past to the present: a review," Progress in Neurobiology, vol. 108, pp. 80-107, 2013.

[14] J. G. Pfaus, G. Damsma, G. G. Nomikos et al., "Sexual behavior enhances central dopamine transmission in the male rat," Brain Research, vol. 530, no. 2, pp. 345-348, 1990.

[15] E. M. Hull, R. C. Eaton, J. Moses, and D. Lorrain, "Copulation increases dopamine activity in the medial preoptic area of male rats," Life Sciences, vol. 52, no. 11, pp. 935-940, 1993.

[16] E. M. Hull, J. Du, D. S. Lorrain, and L. Matuszewich, "Extracellular dopamine in the medial preoptic area: implications for sexual motivation and hormonal control of copulation," Journal of Neuroscience, vol. 15, no. 11, pp. 7465-7471, 1995.

[17] Y. Sato, H. Wada, H. Horita et al., "Dopamine release in the medial preoptic area during male copulatory behavior in rats," Brain Research, vol. 692, no. 1-2, pp. 66-70, 1995.

[18] F. Giuliano and O. Rampin, "Central neural regulation of penile erection," Neuroscience \& Biobehavioral Reviews, vol. 24, no. 5, pp. 517-533, 2000.

[19] T. G. Waddell and D. M. Ibach, "Modern chemical aphrodisiacs," Indian Journal of Pharmaceutical Sciences, vol. 51, no. 3, pp. 79-82, 1989.

[20] S. Moncada, R. M. J. Palmer, and E. A. Higgs, "Nitric oxide: physiology, pathophysiology, and pharmacology," Pharmacological Reviews, vol. 43, no. 2, pp. 109-142, 1991.

[21] S. H. Snyder, "Nitric oxide: first in a new class of neurotransmitters?” Science, vol. 257, no. 5069, pp. 494-496, 1992.

[22] U. Forstermann, L. D. Gorsky, J. S. Pollock, H. H. H. W. Schmidt, M. Heller, and F. Murad, "Regional distribution of EDRF/NO-synthesizing enzyme(s) in rat brain," Biochemical and Biophysical Research Communications, vol. 168, no. 2, pp. 727-732, 1990.

[23] D. S. Bredt, P. M. Hwang, C. E. Glatt, C. Lowenstein, R. R. Reed, and S. H. Snyder, "Cloned and expressed nitric oxide synthase structurally resembles cytochrome P-450 reductase," Nature, vol. 351, no. 6329, pp. 714-718, 1991.

[24] K. E. Anderson, "Pharmacology of lower urinary tract smooth muscles and penile erectile tissues," Pharmacological Reviews, vol. 45, no. 3, pp. 253-308, 1993.

[25] N. Kim, K. M. Azadzoi, I. Goldstein, and I. Saenz de Tejada, “A nitric oxide-like factor mediates nonadrenergic-noncholinergic neurogenic relaxation of penile corpus cavernosum smooth muscle," Journal of Clinical Investigation, vol. 88, no. 1, pp. 112118, 1991.

[26] F. Holmquist, H. Hedlund, and K. E. Andersson, "L-N ${ }^{G}$-nitro arginine inhibits non-adrenergic, non-cholinergic relaxation of human isolated corpus cavernosum," Acta Physiologica Scandinavica, vol. 141, no. 3, pp. 441-442, 1991.

[27] A. Nehra, F. Colreavy, B. K. Khandheria, and K. Chandrasekaran, "Sildenafil citrate, a selective phosphodiesterase type 5 inhibitor: urologic and cardiovascular implications," World Journal of Urology, vol. 19, no. 1, pp. 40-45, 2001.

[28] F. I. Achike and C. Y. Kwan, "Nitric oxide, human diseases and the herbal products that affect the nitric oxide signalling pathway," Clinical and Experimental Pharmacology and Physiology, vol. 30, no. 9, pp. 605-615, 2003.

[29] G. R. Dohle, M. Smit, and R. F. A. Weber, "Androgens and male fertility," World Journal of Urology, vol. 21, no. 5, pp. 341-345, 2003.

[30] P. J. Snyder, "Clinical use of androgens," Annual Review of Medicine, vol. 35, pp. 207-217, 1984.

[31] E. M. Hull, J. Du, D. S. Lorrain, and L. Matuszewich, "Testosterone, preoptic dopamine, and copulation in male rats," Brain Research Bulletin, vol. 44, no. 4, pp. 327-333, 1997.

[32] P. Zvara, R. Sioufi, H. M. Schipper, L. R. Begin, and G. B. Brock, "Nitric oxide mediated erectile activity is a testosterone dependent event: a rat erection model," International Journal of Impotence Research, vol. 7, no. 4, pp. 209-219, 1995.

[33] K. Gauthaman and P. G. Adaikan, "Effect of Tribulus terrestris on nicotinamide adenine dinucleotide phosphate-diaphorase activity and androgen receptors in rat brain," Journal of Ethnopharmacology, vol. 96, no. 1-2, pp. 127-132, 2005.

[34] J. Du and E. M. Hull, "Effects of testosterone on neuronal nitric oxide synthase and tyrosine hydroxylase," Brain Research, vol. 836, no. 1-2, pp. 90-98, 1999.

[35] Y. C. Liu and B. D. Sachs, "Erectile function in male rats after lesions in the lateral paragigantocellular nucleus," Neuroscience Letters, vol. 262, no. 3, pp. 203-206, 1999.

[36] C. Tocharus, R. Jeenapongsa, T. Teakthong, and Y. Smitasiri, "Effects of long-term treatment of Butea superba on sperm motility and concentration," Naresuan University Journal, vol. 13, pp. 11-17, 2005.

[37] A. Manosroi, K. Sanphet, S. Saowakon, S. Aritajat, and J. Manosroi, "Effects of Butea superba on reproductive systems of rats," Fitoterapia, vol. 77, no. 6, pp. 435-438, 2006.

[38] W. Cherdshewasart, P. Bhuntaku, R. Panriansaen, W. Dahlan, and S. Malaivijitnond, "Androgen disruption and toxicity tests of Butea superba Roxb., a traditional herb used for treatment of erectile dysfunction, in male rats," Maturitas, vol. 60, no. 2, pp. 131-137, 2008.

[39] W. Cherdshewasart and N. Nimsakul, "Clinical trial of Buten superba, an alternative herbal treatment for erectile dysfunction," Asian Journal of Andrology, vol. 5, no. 3, pp. 243-246, 2003.

[40] C. Tocharus, Y. Smitasiri, and R. Jeenapongsa, "Butea superba Roxb. enhances penile erection in rats," Phytotherapy Research, vol. 20, no. 6, pp. 484-489, 2006.

[41] N. S. Chauhan and V. K. Dixit, "Antihyperglycemic activity of the ethanolic extract of Curculigo orchioides Gaertn," Pharmacognosy Magazine, vol. 3, pp. 237-240, 2007.

[42] N. S. Chauhan, C. V. Rao, and V. K. Dixit, "Effect of Curculigo orchioides rhizomes on sexual behaviour of male rats," Fitoterapia, vol. 78, no. 7-8, pp. 530-534, 2007.

[43] N. S. Chauhan and V. K. Dixit, "Spermatogenic activity of rhizomes of Curculigo orchioides gaertn in male rats," International Journal of Applied Research in Natural Products, vol. 1, no. 2, pp. 26-31, 2008.

[44] M. Thakur and V. K. Dixit, "Effect of some vajikaran herbs on pendiculation activities and in vitro sperm count in male," Sexuality and Disability, vol. 25, no. 4, pp. 203-207, 2007.

[45] M. Thakur, N. S. Chauhan, S. Bhargava, and V. K. Dixit, "A comparative study on aphrodisiac activity of some ayurvedic herbs in male albino rats," Archives of Sexual Behavior, vol. 38, no. 6, pp. 1009-1015, 2009. 
[46] M. Thakur, R. Loeppert, W. Praznik, and V. K. Dixit, "Effect of some ayurvedic vajikarana rasayana herbs on heat induced testicular damage in male rats," Journal of Complementary and Integrative Medicine, vol. 5, pp. 1-14, 2008.

[47] A. M. Ageel, J. S. Mossa, M. Tariq, M. A. Al-Yahya, and M. S. Al- Said, Saudi Plants Used in Folk Medicine, Department of Scientific Research, King Abdel-Aziz City for Science and Technology, Riyadh, Saudi Arabia, 1987.

[48] H. A. Abd El-Rahman, A. A. El-Badry, O. M. Mahmoud, and F. A. Harraz, "The effect of the aqueous extract of Cynomorium coccineum on the epididymal sperm pattern of the rat," Phytotherapy Research, vol. 13, pp. 248-250, 1999.

[49] E. M. Abdel-Magied, H. A. Abdel-Rahman, and F. M. Harraz, "The effect of aqueous extracts of Cynomorium coccineum and Withania somnifera on testicular development in immature Wistar rats," Journal of Ethnopharmacology, vol. 75, no. 1, pp. $1-4,2001$.

[50] M. Thakur and V. K. Dixit, "A review on some important medicinal plants of Chlorophytum spp," Pharmacognosy Reviews, vol. 2, no. 3, pp. 168-172, 2008.

[51] M. Thakur and V. K. Dixit, "Effect of Chlorophytum borivilianum on androgenic \& sexual behavior of male rats," Indian Drugs, vol. 43, no. 4, pp. 300-306, 2006.

[52] R. Kenjale, R. Shah, and S. Sathaye, "Effects of Chlorophytum borivilianum on sexual behaviour and sperm count in male rats," Phytotherapy Research, vol. 22, no. 6, pp. 796-801, 2008.

[53] M. Thakur, S. Bhargava, W. Praznik, R. Loeppert, and V. K. Dixit, "Effect of Chlorophytum borivilianum Santapau and Fernandes on sexual dysfunction in hyperglycemic male rats," Chinese Journal of Integrative Medicine, vol. 15, no. 6, pp. 448453, 2009.

[54] N. S. Vyawahare, V. G. Kagathara, A. D. Kshirsagar et al., "Effect of hydroalcoholic extract of Chlorophytum borivilianum tubers in alleviating the diabetic impotency in streptozotocin induced male diabetic rats," Pharmacognosy Research, vol. 1, pp. 314-319, 2009.

[55] “The state pharmacopoeia commission of PR China," in Pharmacopoeia of The People's Republic of China English Edition, vol. 1, Chemical Industry Press, Beijing, China, 2000.

[56] M. N. Makarova, O. N. Pozharitskaya, A. N. Shikov, S. V. Tesakova, V. G. Makarov, and V. P. Tikhonov, "Effect of lipidbased suspension of Epimedium koreanum Nakai extract on sexual behavior in rats," Journal of Ethnopharmacology, vol. 114, pp. 412-416, 2007.

[57] W. Liu, Z. Xin, H. Xin, Y. Yuan, L. Tian, and Y. Guo, "Effects of icariin on erectile function and expression of nitric oxide synthase isoforms in castrated rats," Asian Journal of Andrology, vol. 7, no. 4, pp. 381-388, 2005.

[58] H. Ning, Z. C. Xin, G. Lin, L. Banie, T. F. Lue, and C. S. Lin, "Effects of icariin on phosphodiesterase-5 activity in vitro and cyclic guanosine monophosphate level in cavernous smooth muscle cells," Urology, vol. 68, no. 6, pp. 1350-1354, 2006.

[59] Z. Jiang, B. Hu, J. Wang et al., "Effect of icariin on cyclic GMP levels and on the mRNA expression of cGMP-binding cGMPspecific phosphodiesterase (PDE5) in penile cavernosum," Journal of Huazhong University of Science and Technology., vol. 26, no. 4, pp. 460-462, 2006.

[60] M. Dell'Agli, G. V. Galli, E. Dal Cero et al., "Potent inhibition of human phosphodiesterase- 5 by icariin derivatives," Journal of Natural Products, vol. 71, no. 9, pp. 1513-1517, 2008.

[61] H. B. Xu and Z. Q. Huang, "Icariin enhances endothelial nitricoxide synthase expression on human endothelial cells in vitro," Vascular Pharmacology, vol. 47, no. 1, pp. 18-24, 2007.
[62] Z. B. Zhang and Q. T. Yang, "The testosterone mimetic properties of icariin," Asian Journal of Andrology, vol. 8, no. 5, pp. 601-605, 2006.

[63] D. Cyranoski, "Malaysian researchers bet big on home-grown Viagra," Nature Medicine, vol. 11, article 912, 2005.

[64] H. H. Ang and M. K. Sim, "Eurycoma longifolia increases sexual motivation in sexually naive male rats," Archives of Pharmacal Research, vol. 21, no. 6, pp. 779-781, 1998.

[65] H. H. Ang and M. K. Sim, "Eurycoma longifolia Jack enhances libido in sexually experienced male rats," Experimental Animals, vol. 46, no. 4, pp. 287-290, 1997.

[66] H. H. Ang and H. S. Cheang, "Effects of Eurycoma longifolia Jack on laevator ani muscle in both uncastrated and testosteronestimulated castrated intact male rats," Archives of Pharmacal Research, vol. 24, no. 5, pp. 437-440, 2001.

[67] H. H. Ang, S. Ikeda, and E. K. Gan, "Evaluation of the potency activity of aphrodisiac in Eurycoma longifolia Jack," Phytotherapy Research, vol. 15, no. 5, pp. 435-436, 2001.

[68] H. H. Ang and T. H. Ngai, "Aphrodisiac evaluation in noncopulator male rats afterchronic administration of Eurycoma longifolia Jack," Fundamental and Clinical Pharmacology, vol. 15, no. 4, pp. 265-268, 2001.

[69] H. H. Ang and K. L. Lee, "Effect of Eurycoma longifolia Jack on orientation activities in middle-aged male rats," Fundamental and Clinical Pharmacology, vol. 16, no. 6, pp. 479-483, 2002.

[70] H. H. Ang, T. H. Ngai, and T. H. Tan, "Effects of Eurycoma longifolia Jack on sexual qualities in middle aged male rats," Phytomedicine, vol. 10, no. 6-7, pp. 590-593, 2003.

[71] A. F. G. Cicero, S. Piacente, A. Plaza, E. Sala, R. Arletti, and C. Pizza, "Hexanic Maca extract improves rat sexual performance more effectively than methanolic and chloroformic Maca extracts," Andrologia, vol. 34, no. 3, pp. 177-179, 2002.

[72] B. L. Zheng, K. He, C. H. Kim et al., "Effect of a lipidic extract from Lepidium meyenii on sexual behavior in mice and rats," Urology, vol. 55, no. 4, pp. 598-602, 2000.

[73] A. Lentz, K. Gravitt, C. C. Carson, L. Marson, and F. Giuliano, "Acute and chronic dosing of lepidium meyenii (Maca) on male rat sexual behavior," Journal of Sexual Medicine, vol. 4, no. 2, pp. 332-340, 2007.

[74] C. Gonzales, J. Rubio, M. Gasco, J. Nieto, S. Yucra, and G. F. Gonzales, "Effect of short-term and long-term treatments with three ecotypes of Lepidium meyenii (MACA) on spermatogenesis in rats," Journal of Ethnopharmacology, vol. 103, no. 3, pp. 448-454, 2006.

[75] J. Rubio, M. I. Riqueros, M. Gasco, S. Yucra, S. Miranda, and G. F. Gonzales, "Lepidium meyenii (Maca) reversed the lead acetate induced-damage on reproductive function in male rats," Food and Chemical Toxicology, vol. 44, no. 7, pp. 1114-1122, 2006.

[76] S. Yucra, M. Gasco, J. Rubio, J. Nieto, and G. F. Gonzales, "Effect of different fractions from hydroalcoholic extract of Black Maca (Lepidium meyenii) on testicular function in adult male rats," Fertility and Sterility, vol. 89, no. 5, pp. 1461-1467, 2008.

[77] G. F. Gonzalez, A. Córdova, K. Vega, A. Chung, A. Villena, and C. Góñez, "Effect of Lepidium meyenii (Maca), a root with aphrodisiac and fertility-enhancing propeties, on serum reproductive hormone levels in adult healthy men," Journal of Endocrinology, vol. 176, no. 1, pp. 163-168, 2003.

[78] A. C. Ruiz-Luna, S. Salazar, N. J. Aspajo, J. Rubio, M. Gasco, and G. F. Gonzales, "Lepidium meyenii (Maca) increases litter size in normal adult female mice," Reproductive Biology and Endocrinology, vol. 3, article 16, 2005.

[79] G. F. Gonzales, A. Cordova, C. Gonzales, A. Chung, K. Vega, and A. Villena, "Lepidium meyenii (Maca) improved semen 
parameters in adult men," Asian Journal of Andrology, vol. 3, no. 4, pp. 301-303, 2001.

[80] G. F. Gonzales, A. Córdova, K. Vega et al., "Effect of Lepidium meyenii (MACA) on sexual desire and its absent relationship with serum testosterone levels in adult healthy men," Andrologia, vol. 34, no. 6, pp. 367-372, 2002.

[81] P. Bogani, F. Simonini, M. Iriti et al., "Lepidium meyenii (Maca) does not exert direct androgenic activities," Journal of Ethnopharmacology, vol. 104, no. 3, pp. 415-417, 2006.

[82] G. F. Gonzales, S. Miranda, J. Nieto et al., "Red maca (Lepidium meyenii) reduced prostate size in rats.", eproductive Biology and Endocrinology, vol. 3, article 5, 2005.

[83] F. Chung, J. Rubio, C. Gonzales, M. Gasco, and G. F. Gonzales, "Dose-response effects of Lepidium meyenii (Maca) aqueous extract on testicular function and weight of different organs in adult rats," Journal of Ethnopharmacology, vol. 98, no. 1-2, pp. 143-147, 2005.

[84] S. Saksena and V. K. Dixit, "Role of total alkaloids of Mucuna pruriens Baker in spermatogenesis in Albino rats," Indian Journal of Natural Products, vol. 3, pp. 3-7, 1987.

[85] Y. M. N. Amin, Z. S. Rehman, and N. A. Khan, "Sexual function improving effect of M. pruriens in sexually normal male rats," Fitoterapial, vol. 67, pp. 53-58, 1996.

[86] A. K. V. Kumar and K. K. Srinivasan, "Aphrodisiac activity of the seeds of Mucuna pruriens," Indian Drugs, vol. 31, no. 7, pp. 321-327, 1994.

[87] S. Suresh, E. Prithiviraj, and S. Prakash, "Effect of Mucuna pruriens on oxidative stress mediated damage in aged rat sperm," International Journal of Andrology, vol. 33, no. 1, pp. 2232, 2010.

[88] A. P. Singh, S. Sarkar, M. Tripathi, and S. Rajender, "Mucuna pruriens and its major constituent L-DOPA recover spermatogenic loss by combating ROS, loss of mitochondrial membrane potential and apoptosis," PLoS ONE, vol. 8, no. 1, Article ID e54655, 2013.

[89] S. Suresh and S. Prakash, "Effect of Mucuna pruriens (Linn.) on sexual behavior and sperm parameters in streptozotocininduced diabetic male rat," Journal of Sexual Medicine, vol. 9, no. 12, pp. 3066-3078, 2012.

[90] M. K. Ahmad, A. A. Mahdi, K. K. Shukla, N. Islam, S. P. Jaiswar, and S. Ahmad, "Effect of Mucuna pruriens on semen profile and biochemical parameters in seminal plasma of infertile men," Fertility and Sterility, vol. 90, no. 3, pp. 627-635, 2008.

[91] K. K. Shukla, A. A. Mahdi, S. N. Shankwar, and M. K. Ahmad, "Effect of Mucuna pruriens on hormonal status and semen quality in infertile males," Contraception, vol. 78, p. 194, 2008.

[92] K. K. Shukla, A. A. Mahdi, M. K. Ahmad, S. N. Shankhwar, S. Rajender, and S. P. Jaiswar, "Mucuna pruriens improves male fertility by its action on the hypothalamus-pituitary-gonadal axis," Fertility and Sterility, vol. 92, no. 6, pp. 1934-1940, 2009.

[93] M. K. Ahmad, A. A. Mahdi, K. K. Shukla, N. Islam, S. P. Jaiswar, and S. Ahmad, "Effect of Mucuna pruriens on semen profile and biochemical parameters in seminal plasma of infertile men," Fertility and Sterility, vol. 90, no. 3, pp. 627-635, 2008.

[94] K. K. Shukla, A. A. Mahdi, M. K. Ahmad, S. P. Jaiswar, S. N. Shankwar, and S. C. Tiwari, "Mucuna pruriens reduces stress and improves the quality of semen in infertile men," EvidenceBased Complementary and Alternative Medicine, vol. 7, no. 1, pp. 137-144, 2010.

[95] R. N. Chopra, I. C. Chopra, K. L. Handa, and L. D. U. N. Kapoor, Eds., Chopra's Indigenous Drugs of India, Dhur \& Sons Private Ltd., Calcutta, India, 2nd edition, 1958.
[96] P. Georgiev, M. Dimitrov, and S. Vitanov, "Effect of Tribestan (from Tribulus terrestris) on plasma testosterone and spermatogenesis in male lambs and rams," Veterinarna Sbirka, vol. 86, no. 3, pp. 20-22, 1988.

[97] F. Koumanov, E. Bozadjieva, and M. Andreeva, "Clinical trial of the drug Tribestan," Savremenna Medicina, vol. 33, no. 4, pp. 211-215, 1982.

[98] A. Adimoelja and P. G. Adaikan, "Protodioscin from herbal plant Tribulus terrestris L. improves male sexual functions possibly via DHEA," International Journal of Impotence Research, vol. 9, article S6, 1997.

[99] K. Gauthaman, P. G. Adaikan, R. N. V. Prasad, V. H. H. Goh, and S. C. Ng, "Changes in hormonal parameters secondary to intravenous administration of Tribulus terrestris extract in primates," International Journal of Impotence Research, vol. 1, p. 6, 2000.

[100] P. G. Adaikan, K. Gauthaman, R. N. V. Prasad, and S. C. Ng, "Proerectile pharmacological effects of Tribulus terrestris extract on the rabbit corpus cavernosum," Annals of the Academy of Medicine Singapore, vol. 29, no. 1, pp. 22-26, 2000.

[101] K. Gauthaman, P. G. Adaikan, and R. N. V. Prasad, "Aphrodisiac properties of Tribulus Terrestris extract (Protodioscin) in normal and castrated rats," Life Sciences, vol. 71, no. 12, pp. 13851396, 2002.

[102] K. Gauthaman, A. P. Ganesan, and R. N. V. Prasad, "Sexual effects of puncturevine (Tribulus terrestris) extract (protodioscin): an evaluation using a rat model," Journal of Alternative and Complementary Medicine, vol. 9, no. 2, pp. 257-265, 2003.

[103] K. Gauthaman and P. G. Adaikan, "Effect of Tribulus terrestris on nicotinamide adenine dinucleotide phosphate-diaphorase activity and androgen receptors in rat brain," Journal of Ethnopharmacology, vol. 96, no. 1-2, pp. 127-132, 2005.

[104] K. Gauthaman and A. P. Ganesan, "The hormonal effects of Tribulus terrestris and its role in the management of male erectile dysfunction: an evaluation using primates, rabbit and rat," Phytomedicine, vol. 15, no. 1-2, pp. 44-54, 2008.

[105] L. C. Garg and G. C. Parasar, "Effect of Withania somnifera on reproduction in mice," Planta Medica, vol. 13, pp. 46-47, 1965.

[106] I. Ilayperuma, W. D. Ratnasooriya, and T. R. Weerasooriya, "Effect of Withania somnifera root extract on the sexual behaviour of male rats," Asian Journal of Andrology, vol. 4, no. 4, pp. 295-298, 2002.

[107] P. C. Mali, P. S. Chouhan, and R. Chaudhary, "Evaluation of antifertility activity of Withania somnifera in male albino rats," Fertility and Sterility, vol. 90, p. S18, 2008.

[108] D. S. Misra, R. K. Maiti, S. Bera, K. Das, and D. Ghosh, "Protective effect of composite extract of Withania somnifera, Ocimum sanctum and Zingiber officinale on swimming-induced reproductive Endocrine dysfunctions in male rat," Iranian Journal of Pharmacology \& Therapeutics, vol. 4, pp. 110-117, 2005.

[109] T. Iuvone, G. Esposito, F. Capasso, and A. A. Izzo, "Induction of nitric oxide synthase expression by Withania somnifera in macrophages," Life Sciences, vol. 72, no. 14, pp. 1617-1625, 2003.

[110] V. R. Ambiye, D. Langade, S. Dongre, P. Aptikar, M. Kulkarni, and A. Dongre, "Clinical evaluation of the spermatogenic activity of the root extract of Ashwagandha (Withania somnifera) in oligospermic males: a pilot study," Evidence-Based Complementary and Alternative Medicine, vol. 2013, Article ID 571420, 6 pages, 2013.

[111] M. K. Ahmad, A. A. Mahdi, K. K. Shukla et al., "Withania somnifera improves semen quality by regulating reproductive hormone levels and oxidative stress in seminal plasma of 
infertile males," Fertility and Sterility, vol. 94, no. 3, pp. 989-996, 2010.

[112] A. A. Mahdi, K. K. Shukla, M. K. Ahmad et al., "Withania somnifera improves semen quality in stress-related male fertility," Evidence-Based Complementary and Alternative Medicine, vol. 2011, Article ID 576962, 9 pages, 2011.

[113] N. S. Chauhan, D. K. Saraf, and V. K. Dixit, "Effect of vajikaran rasayana herbs on pituitary-gonadal axis," European Journal of Integrative Medicine, vol. 2, no. 2, pp. 89-91, 2010.

[114] P. Kamtchouing, G. Y. F. Mbongue, T. Dimo, P. Watcho, H. B. Jatsa, and S. D. Sokeng, "Effects of Aframomum melegueta and Piper guineense on sexual behaviour of male rats," Behavioural Pharmacology, vol. 13, no. 3, pp. 243-247, 2002.

[115] M. Thakur, S. Bhargava, and V. K. Dixit, "Effect of Asparagus racemosus on sexual dysfunction in hyperglycemic male rats," Pharmaceutical Biology, vol. 47, no. 5, pp. 390-395, 2009.

[116] H. Guohua, L. Yanhua, M. Rengang, W. Dongzhi, M. Zhengzhi, and Z. Hua, "Aphrodisiac properties of Allium tuberosum seeds extract," Journal of Ethnopharmacology, vol. 122, no. 3, pp. 579$582,2009$.

[117] W. D. Ratnasooriya and J. R. Jayakody, "Effects of aqueous extract of Alpinia calcarata rhizomes on reproductive competence of male rats," Acta Biologica Hungarica, vol. 57, no. 1, pp. 23-35, 2006.

[118] A. Subramoniam, V. Madhavachandran, K. Ravi, and V. S. Anuja, "Aphrodisiac property of the elephant creeper Argyreia nervosa," Journal of Endocrinology and Reproduction, vol. 2, pp. 82-85, 2007.

[119] A. R. Campos, R. C. P. Lima Jr., D. E. A. Uchoa, E. R. Silveira, F. A. Santos, and V. S. N. Rao, "Pro-erectile effects of an alkaloidal rich fraction from Aspidosperma ulei root bark in mice," Journal of Ethnopharmacology, vol. 104, no. 1-2, pp. 240-244, 2006.

[120] N. S. Chauhan, V. Sharma, and V. K. Dixit, "Effect of Asteracantha longifolia seeds on the sexual behaviour of male rats," Natural Product Research: Formerly Natural Product Letters, vol. 25, no. 15, pp. 1423-1431, 2011.

[121] V. Sharma, M. Thakur, N. S. Chauhan, and V. K. Dixit, "Evaluation of the anabolic, aphrodisiac and reproductive activity of Anacyclus pyrethrum DC in male rats," Scientia Pharmaceutica, vol. 77, no. 1, pp. 97-110, 2009.

[122] V. Sharma, M. Thakur, N. S. Chauhan, and V. K. Dixit, "Effects of petroleum ether extract of Anacyclus pyrethrum DC. on sexual behavior in male rats," Journal of Chinese Integrative Medicine, vol. 8, no. 8, pp. 767-773, 2010.

[123] V. Sharma, J. Boonen, B. de Spiegeleer, and V. K. Dixit, "Androgenic and spermatogenic activity of alkylamide-rich ethanol solution extract of Anacyclus pyrethrum DC," Phytotherapy Research, vol. 27, no. 1, pp. 99-106, 2013.

[124] L. Tédong, D. P. Djomeni Dzeufiet, T. Dimo et al., "Effect of leaf Anacardium occidentale L. (Anacardiaceae) hexane extract on reproductive function in streptozotocin-induced diabetic rats," Phytotherapie, vol. 5, no. 4, pp. 182-193, 2007.

[125] M. T. Yakubu and A. J. Afolayan, "Effect of aqueous extract of Bulbine natalensis (Baker) stem on the sexual behaviour of male rats," International Journal of Andrology, vol. 32, no. 6, pp. 629636, 2009.

[126] S. Ramachandran, Y. Sridhar, S. K. G. Sam et al., "Aphroidisiac activity of Butea frondosa Koen. ex Roxb. extract in male rats," Phytomedicine, vol. 11, no. 2-3, pp. 165-168, 2004.

[127] N. S. Chauhan and V. K. Dixit, "Effects of Bryonia laciniosa seeds on sexual behaviour of male rats," International Journal of Impotence Research, vol. 22, no. 3, pp. 190-195, 2010.
[128] P. F. Moundipa, N. S. E. Beboy, F. Zelefack et al., "Effects of Basella alba and Hibiscus macranthus extracts on testosterone production of adult rat and bull leydig cells," Asian Journal of Andrology, vol. 7, no. 4, pp. 411-417, 2005.

[129] P. Sudwan, K. Saenphet, S. Aritajat, and N. Sitasuwan, "Effects of Boesenbergia rotunda (L.) Mansf. on sexual behaviour of male rats," Asian Journal of Andrology, vol. 9, no. 6, pp. 849-855, 2007.

[130] S. T. Ali and N. I. Rakkah, "Probable neuro sexual mode of action of Casimiroa edulis seed extract verses sildenafil citrate (viagra) on mating behavior in normal male rats," Pakistan Journal of Pharmaceutical Sciences, vol. 21, no. 1, pp. 1-6, 2008.

[131] W. D. Ratnasooriya and T. S. P. Fernando, "Effect of black tea brew of Camellia sinensis on sexual competence of male rats," Journal of Ethnopharmacology, vol. 118, no. 3, pp. 373-377, 2008.

[132] M. Abdulwaheb, E. Makonnen, A. Debella, and D. Abebe, "Effect of Catha edulis foresk (khat) extracts on male rat sexual behavior," Journal of Ethnopharmacology, vol. 110, no. 2, pp. 250256, 2007.

[133] A. Zamblé, F. Martin-Nizard, S. Sahpaz et al., "Vasoactivity, antioxidant and aphrodisiac properties of Caesalpinia benthamiana roots," Journal of Ethnopharmacology, vol. 116, no. 1, pp. 112-119, 2008.

[134] B. Sangameswaran and B. Jayakar, "Anti-diabetic and spermatogenic activity of Cocculus hirsutus (L) diels," African Journal of Biotechnology, vol. 6, no. 10, pp. 1212-1216, 2007.

[135] M. Thakur, N. S. Chauhan, V. Sharma, V. K. Dixit, and S. Bhargava, "Effect of Curculigo orchioides on hyperglycemiainduced oligospermia and sexual dysfunction in male rats," International Journal of Impotence Research, vol. 24, no. 1, pp. 31-37, 2012.

[136] H. Hosseinzadeh, T. Ziaee, and A. Sadeghi, "The effect of saffron, Crocus sativus stigma, extract and its constituents, safranal and crocin on sexual behaviors in normal male rats," Phytomedicine, vol. 15, no. 6-7, pp. 491-495, 2008.

[137] G. Onuaguluchi and P. Nwafor, "Pharmacological basis for the use of the antivenene water soluble extract of Diodia scandens as a laxative, oxytocic agent and a possible aphrodisiac in traditional medicine practice in eastern Nigeria," Phytotherapy Research, vol. 13, pp. 459-463, 1999.

[138] P. Watcho, M. Wankeu-Nya, T. B. Nguelefack, L. Tapondjou, R. Teponno, and A. Kamanyi, "Pro-sexual effects of Dracaena arborea (wild) link (dracaenaceae) in sexually experienced male rats," Pharmacologyonline, vol. 1, pp. 400-419, 2007.

[139] M. Thakur and V. K. Dixit, "Aphrodisiac activity of Dactylorhiza hatagirea (D.Don) Soo in male albino rats," Evidence-based Complementary and Alternative Medicine, vol. 4, no. 1, pp. 2931, 2007.

[140] S. P. Jagdale, S. Shimpi, and D. Chachad, "Pharmacological studies of 'Salep"' Journal of Herbal Medicine and Toxicology, vol. 3, pp. 153-156, 2009.

[141] J. H. Chiu, K. K. Chen, T. M. Chien et al., "Epimedium brevicornum Maxim extract relaxes rabbit corpus cavernosum through multitargets on nitric oxide/cyclic guanosine monophosphate signaling pathway," International Journal of Impotence Research, vol. 18, no. 4, pp. 335-342, 2006.

[142] M. T. Yakubu, M. A. Akanji, and A. T. Oladiji, "Aphrodisiac potentials of the aqueous extract of Fadogia agrestis (Schweinf. Ex Hiern) stem in male albino rats," Asian Journal of Andrology, vol. 7, no. 4, pp. 399-404, 2005.

[143] P. Zanoli, A. Benelli, M. Rivasi, C. Baraldi, F. Vezzalini, and M. Baraldi, "Opposite effect of acute and subchronic treatments with Ferula hermonis on copulatory behavior of male rats," 
International Journal of Impotence Research, vol. 15, no. 6, pp. 450-455, 2003.

[144] K. A. Oluyemi, O. R. Jimoh, O. A. Adesanya, I. O. Omotuyi, S. J. Josiah, and T. O. Oyesola, "Effects of crude ethanolic extract of Garcinia cambogia on the reproductive system of male wistar rats (Rattus novergicus)," African Journal of Biotechnology, vol. 6, no. 10, pp. 1236-1238, 2007.

[145] A. Amin and A. E. A. Hamza, "Effects of Roselle and Ginger on cisplatin-induced reproductive toxicity in rats," Asian Journal of Andrology, vol. 8, no. 5, pp. 607-612, 2006.

[146] G. Chaturapanich, S. Chaiyakul, V. Verawatnapakul, and C. Pholpramool, "Effects of Kaempferia parviflora extracts on reproductive parameters and spermatic blood flow in male rats," Reproduction, vol. 136, no. 4, pp. 515-522, 2008.

[147] G. Chaturapanich, S. Chaiyakul, V. Verawatnapakul, T. Yimlamai, and C. Pholpramool, "Enhancement of aphrodisiac activity in male rats by ethanol extract of Kaempferia parviflora and exercise training," Andrologia, vol. 44, supplement 1, pp. 323328, 2012.

[148] E. Etuk and A. A. Muhammad, "Safety evaluations of aqueous stem bark extract of Lophira lanceolata in sprague dawley rats," International Journal of Research in Pharmaceutical Sciences, vol. 1, no. 1, pp. 28-33, 2010.

[149] J. Ilarionov, "Androgenic and aphrodisiac action of medical plant Lithospermum arvense (bird millet)," Eksperimentalna Medicina I Morfologiya, vol. 28, no. 1, pp. 28-33, 1989.

[150] M. T. Yakubu, M. A. Akanji, A. T. Oladiji, and A. A. Adesokan, "Androgenic potentials of aqueous extract of Massularia acuminata (G. Don) Bullock ex Hoyl. stem in male Wistar rats," Journal of Ethnopharmacology, vol. 118, no. 3, pp. 508-513, 2008.

[151] P. Watcho, P. Kamtchouing, S. D. Sokeng et al., "Androgenic effect of Mondia whitei roots in male rats," Asian Journal of Andrology, vol. 6, no. 3, pp. 269-272, 2004.

[152] F. Lampiao, D. Krom, and S. S. Du Plessis, "The in vitro effects of Mondia whitei on human sperm motility parameters," Phytotherapy Research, vol. 22, no. 9, pp. 1272-1273, 2008.

[153] A. Zamblé, S. Sahpaz, C. Brunet, and F. Bailleul, "Effects of keayana on sexual behavior of male rats," Phytomedicine, vol. 15 , no. 8, pp. 625-629, 2008.

[154] M. Carro-Juárez, E. Cervantes, M. Cervantes-Méndez, and G. Rodríguez-Manzo, "Aphrodisiac properties of Montanoa tomentosa aqueous crude extract in male rats," Pharmacology Biochemistry and Behavior, vol. 78, no. 1, pp. 129-134, 2004.

[155] A. Tajuddin, S. Ahmad, A. Latif, and I. A. Qasmi, "Aphrodisiac activity of $50 \%$ ethanolic extracts of Myristica fragrans Houtt. (Nutmeg) and Syzygium aromaticum (L.) Merr. \& Perry. (Clove) in male mice: a comparative study," BMC Complementary and Alternative Medicine, vol. 3, article 6, 2003.

[156] S. Ahmad, A. Latif, I. A. Qasmi, and K. M. Y. Amin, "An experimental study of sexual function improving effect of Myristica fragrans Houtt. (Nutmeg)," BMC Complementary and Alternative Medicine, vol. 5, article 16, 2005.

[157] M. Thakur and V. K. Dixit, "Ameliorative effect of fructooligosaccharide rich extract of Orchis latifolia linn. on sexual dysfunction in hyperglycemic male rats," Sexuality and Disability, vol. 26, no. 1, pp. 37-46, 2008.

[158] L. L. Murphy and T. J. Lee, "Ginseng, sex behavior, and nitric oxide," Annals of the New York Academy of Sciences, vol. 962, pp. 372-377, 2002.

[159] L. L. Murphy, R. S. Cadena, D. Chávez, and J. S. Ferraro, "Effect of American ginseng (Panax quinquefolium) on male copulatory behavior in the rat," Physiology and Behavior, vol. 64, no. 4, pp. 445-450, 1998.
[160] A. J. Riley, "Yohimbine in the treatment of erectile disorder," British Journal of Clinical Practice, vol. 48, no. 3, pp. 133-136, 1994.

[161] F. Subhan, S. Sultan, W. Alam, and A. S. T. Dil, "Aphrodisiac potential of Peganum harmala seeds," Hamdard Medicus, vol. 41, pp. 69-72, 1998.

[162] P. Kamtchouing, G. Y. M. Fandio, T. Dimo, and H. B. Jatsa, "Evaluation of androgenic activity of Zingiber officinale and Pentadiplandra brazzeana in male rats," Asian Journal of Andrology, vol. 4, no. 4, pp. 299-301, 2002.

[163] M. Oshima and Y. Gu, "Pfaffia paniculata-induced changes in plasma estradiol-17 $\beta$, progesterone and testosterone levels in mice," Journal of Reproduction and Development, vol. 49, no. 2, pp. 175-180, 2003.

[164] O. B. Akinola, O. S. Oladosu, and O. O. Dosumu, "Ethanol extract of the leaves of Psidium guajava Linn enhances sperm output in healthy Wistar rats," African Journal of Medicine and Medical Sciences, vol. 36, no. 2, pp. 137-140, 2007.

[165] W. M. Yang, M. S. Chang, and S. K. Park, "Effects of Psoralea corylifolia on the cAMP-responsive element modulator (CREM) expression and spermatogenesis in rats," Journal of Ethnopharmacology, vol. 117, no. 3, pp. 503-506, 2008.

[166] K. Dhawan, S. Kumar, and A. Sharma, "Aphrodisiac activity of methanol extract of leaves of Passiflora incarnata Linn. in mice," Phytotherapy Research, vol. 17, no. 4, pp. 401-403, 2003.

[167] M. S. Oh, W. M. Yang, M. S. Chang et al., "Effects of Rubus coreanus on sperm parameters and CAMP-responsive element modulator (CREM) expression in rat testes," Journal of Ethnopharmacology, vol. 114, no. 3, pp. 463-467, 2007.

[168] N. C. Rakuambo, J. J. M. Meyer, A. Hussein, C. Huyser, S. P. Mdlalose, and T. G. Raidani, "In vitro effect of medicinal plants used to treat erectile dysfunction on smooth muscle relaxation and human sperm," Journal of Ethnopharmacology, vol. 105, no. 1-2, pp. 84-88, 2006.

[169] A. Tajuddin, S. Ahmad, A. Latif, and I. A. Qasmi, "Effect of $50 \%$ ethanolic extract of Syzygium aromaticum (L.) Merr. \& Perry. (clove) on sexual behaviour of normal male rats," BMC Complementary and Alternative Medicine, vol. 4, article 17, 2004.

[170] M. Carro-Juárez, C. Alcazar, E. Ballesteros-Polvo, and P. Villalobos-Peñalosa, "Increase of ejaculatory capacity by systemic administration of the oquichpatli (Senecio cardiophyllus) aqueous crude extract in male rats," Journal of Ethnopharmacology, vol. 126, no. 3, pp. 506-511, 2009.

[171] M. W. Islam, M. Tariq, A. M. Ageel, M. S. Al-Said, and A. M. AlYhya, "Effect of Salvia haematodes on sexual behavior of male rats," Journal of Ethnopharmacology, vol. 33, no. 1-2, pp. 67-72, 1991.

[172] W. D. Ratnasooriya and M. G. Dharmasiri, "Effects of Terminalia catappa seeds on sexual behaviour and fertility of male rats," Asian Journal of Andrology, vol. 2, no. 3, pp. 213-219, 2000.

[173] W. H. A. El-Tantawy, A. Temraz, and O. D. El-Gindi, "Free serum testosterone level in male rats treated with Tribulus alatus extracts," International Brazilian Journal of Urology, vol. 33, no. 4, pp. 554-559, 2007.

[174] A. Subramoniam, V. Madhavachandran, S. Rajasekharan, and P. Pushpangadan, "Aphrodisiac property of Trichopus zeylanicus extract in male mice," Journal of Ethnopharmacology, vol. 57, no. 1, pp. 21-27, 1997.

[175] S. A. Padashetty and S. H. Mishra, "Aphrodisiac studies of Tricholepis glaberrima with supportive action from antioxidant enzymes," Pharmaceutical Biology, vol. 45, no. 7, pp. 580-586, 2007. 
[176] R. Arletti, A. Benelli, E. Cavazzuti, G. Scarpetta, and A. Bertolini, "Stimulating property of Turnera diffusa and Pfaffia paniculata extracts on the sexual behavior of male rats," Psychopharmacology, vol. 143, no. 1, pp. 15-19, 1999.

[177] P. K. Suresh Kumar, A. Subramoniam, and P. Pushpangadan, "Aphrodisiac activity of Vanda tessellata (Roxb.) Hook. ex Don extract in male mice," Indian Journal of Pharmacology, vol. 32, no. 5, pp. 300-304, 2000.

[178] N. S. Chauhan, V. Sharma, M. Thakur, A. C. H. F. Sawaya, and V. K. Dixit, "Pueraria tuberosa DC extract improves androgenesis and sexual behavior via FSH LH cascade," The Scientific World Journal, vol. 2013, Article ID 780659, 8 pages, 2013.

[179] V. Sharma, J. Boonen, N. S. Chauhan, M. Thakur, B. de Spiegeleer, and V. K. Dixit, "Spilanthes acmella ethanolic flower extract: LC-MS alkylamide profiling and its effects on sexual behavior in male rats," Phytomedicine, vol. 18, no. 13, pp. 11611169, 2011.

[180] V. Sharma, M. Thakur, and V. K. Dixit, "A comparative study of ethanolic extracts of Pedalium murex Linn. fruits and sildenafil citrate on sexual behaviors and serum testosterone level in male rats during and after treatment," Journal of Ethnopharmacology, vol. 143, no. 1, pp. 201-206, 2012.

[181] P. Watcho, W. N. Modeste, K. Albert, and M. Carro-Juarez, "Dracaena arborea extracts delay the pro-ejaculatory effect of dopamine and oxytocin in spinal male rats," International Journal of Impotence Research, 2014.

[182] A. K. Sanda, P. Miegueu, D. C. Bilanda et al., "Ejaculatory activities of Allanblackia floribunda stem bark in spinal male rats," Pharmaceutical Biology, vol. 51, no. 8, pp. 1014-1020, 2013.

[183] S. Kataria, D. Kaur, S. K. Rao, and R. K. Khajuria, "In vitro and in vivo aphrodisiac properties of Corchorus depressus Linn. on rabbit corpus cavernosum smooth muscle relaxation and sexual behavior of normal male rats," Journal of Ethnopharmacology, vol. 148, no. 1, pp. 210-217, 2013.

[184] C. JianFeng, Z. PengYing, X. ChengWei, H. TaoTao, B. YunGui, and C. KaoShan, "Effect of aqueous extract of Arctium lappa L. (burdock) roots on the sexual behavior of male rats," $B M C$ Complementary and Alternative Medicine, vol. 12, article 8, 2012.

[185] M. T. Yakubu, T. O. Oyeyipo, A. L. Quadri, and M. A. Akanji, "Effects of aqueous extract of Musa paradisiaca root on testicular function parameters of male rats," Journal of Basic and Clinical Physiology and Pharmacology, vol. 24, no. 2, pp. 151-157, 2013.

[186] T. O. Ajiboye, Q. O. Nurudeen, and M. T. Yakubu, "Aphrodisiac effect of aqueous root extract of Lecaniodiscus cupanioides in sexually impaired rats," Journal of Basic and Clinical Physiology and Pharmacology, vol. 25, no. 2, pp. 241-248, 2014.

[187] S. Srisuwan, J. Sattayasai, T. Arkaravichien, and C. Aromdee, "Effects of andrographolide and its semisynthetic derivative on sexual behaviors in male mice," Thai Journal of Pharmacology, vol. 31, pp. 68-71, 2009.

[188] S. A. Taha, A. M. Ageel, M. W. Islam, and O. T. Ginawi, "Effect of (-)-cathinone, a psychoactive alkaloid from khat (Catha edulis forsk.) and caffeine on sexual behaviour in rats," Pharmacological Research, vol. 31, no. 5, pp. 299-303, 1995.

[189] P. Zanoli, M. Rivasi, M. Zavatti, F. Brusiani, F. Vezzalini, and M. Baraldi, "Activity of single components of Ferula hermonis on male rat sexual behavior," International Journal of Impotence Research, vol. 17, no. 6, pp. 513-518, 2005.

[190] T. Colman-Saizarbitoria, P. Boutros, A. Amesty et al., "Ferutinin stimulates nitric oxide synthase activity in median eminence of the rat," Journal of Ethnopharmacology, vol. 106, no. 3, pp. 327332, 2006.
[191] X. Wang, S. Chu, T. Qian, J. Chen, and J. Zhang, "Ginsenoside Rg1 improves male copulatory behavior via nitric oxide/ cyclic guanosine monophosphate pathway," Journal of Sexual Medicine, vol. 7, no. 2, pp. 743-750, 2010.

[192] Q. Luo, Z. Li, X. Huang, J. Yan, S. Zhang, and Y. Cai, "Lycium barbarum polysaccharides: Protective effects against heat-induced damage of rat testes and $\mathrm{H}_{2} \mathrm{O}_{2}$-induced DNA damage in mouse testicular cells and beneficial effect on sexual behavior and reproductive function of hemicastrated rats," Life Sciences, vol. 79, no. 7, pp. 613-621, 2006.

[193] L. Taepongsorat, P. Tangpraprutgul, N. Kitana, and S. Malaivijitnond, "Stimulating effects of quercetin on sperm quality and reproductive organs in adult male rats," Asian Journal of Andrology, vol. 10, no. 2, pp. 249-258, 2008.

[194] B. B. Benson, Y. A. BéKro, J. A. MamyrbéKova-BéKro, W. K. Coulibaly, and E. E. Ehilé, "Assessment of sexual stimulant potential of total flavonoids extracted from leaves of Palisota hirsuta Thunb. K. Schum (Commelinaceae)," European Journal of Scientific Research, vol. 22, no. 4, pp. 533-538, 2008.

[195] M. Sala, D. Braida, M. P. Leone, P. Calcaterra, S. Monti, and E. Gori, "Central effect of yohimbine on sexual behavior in the rat," Physiology \& Behavior, vol. 47, no. 1, pp. 165-173, 1990.

[196] R. H. Peters, P. C. Koch, and B. L. Blythe, "Differential effects of yohimbine and naloxone on copulatory behaviors of male rats," Behavioral Neuroscience, vol. 102, no. 4, pp. 559-564, 1988.

[197] J. J. M. Meyer, N. C. Rakuambo, and A. A. Hussein, "Novel xanthones from Securidaca longepedunculata with activity against erectile dysfunction," Journal of Ethnopharmacology, vol. 119, no. 3, pp. 599-603, 2008.

[198] S. Haeri, B. Minaie, G. Amin et al., "Effect of Satureja khuzestanica essential oil on male rat fertility," Fitoterapia, vol. 77, no. 7-8, pp. 495-499, 2006.

[199] R. M. Tyagi, U. M. Aswar, V. Mohan, S. L. Bodhankar, G. N. Zambare, and P. A. Thakurdesai, "Study of furostenol glycoside fraction of Tribulus terresteris on male sexual function in rats," Pharmaceutical Biology, vol. 46, no. 3, pp. 191-198, 2008.

[200] R. Estrada-Reyes, P. Ortiz-López, J. Gutiérrez-Ortíz, and L. Martínez-Mota, "Turnera diffusa Wild (Turneraceae) recovers sexual behavior in sexually exhausted males," Journal of Ethnopharmacology, vol. 123, no. 3, pp. 423-429, 2009.

[201] A. Subramoniam, A. Gangaprasad, P. K. Sureshkumar, J. Radhika, and B. K. Arun, "A novel aphrodisiac compound from an orchid that activates nitric oxide synthases," International Journal of Impotence Research, vol. 25, no. 6, pp. 212-216, 2013.

[202] J. S. Park and K. Han, "The spermatogenic effect of Yacon extract and its constituents and their inhibition effect of testosterone metabolism," Biomolecules and Therapeutics, vol. 21, no. 2, pp. 153-160, 2013. 

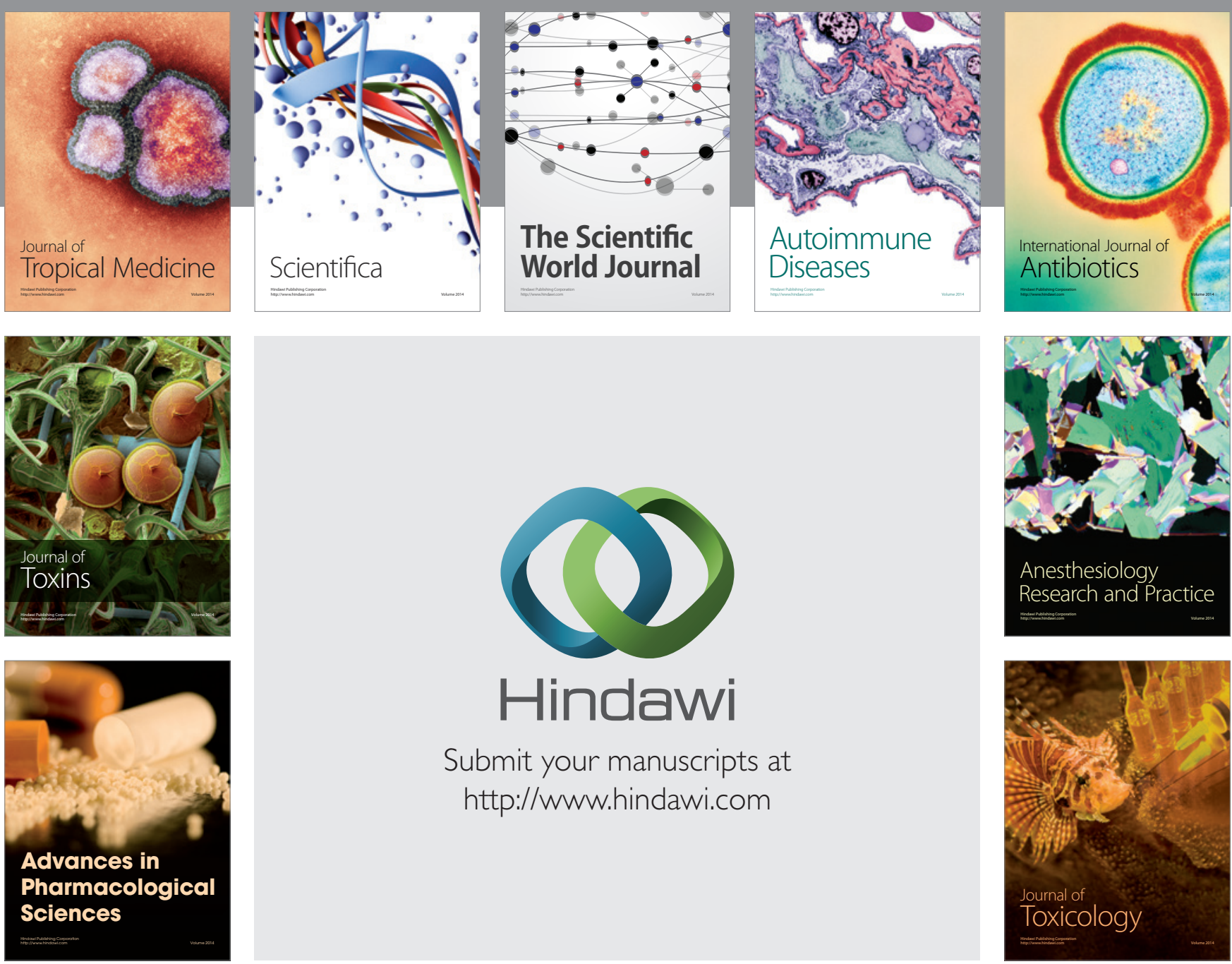

\section{Hindawi}

Submit your manuscripts at

http://www.hindawi.com
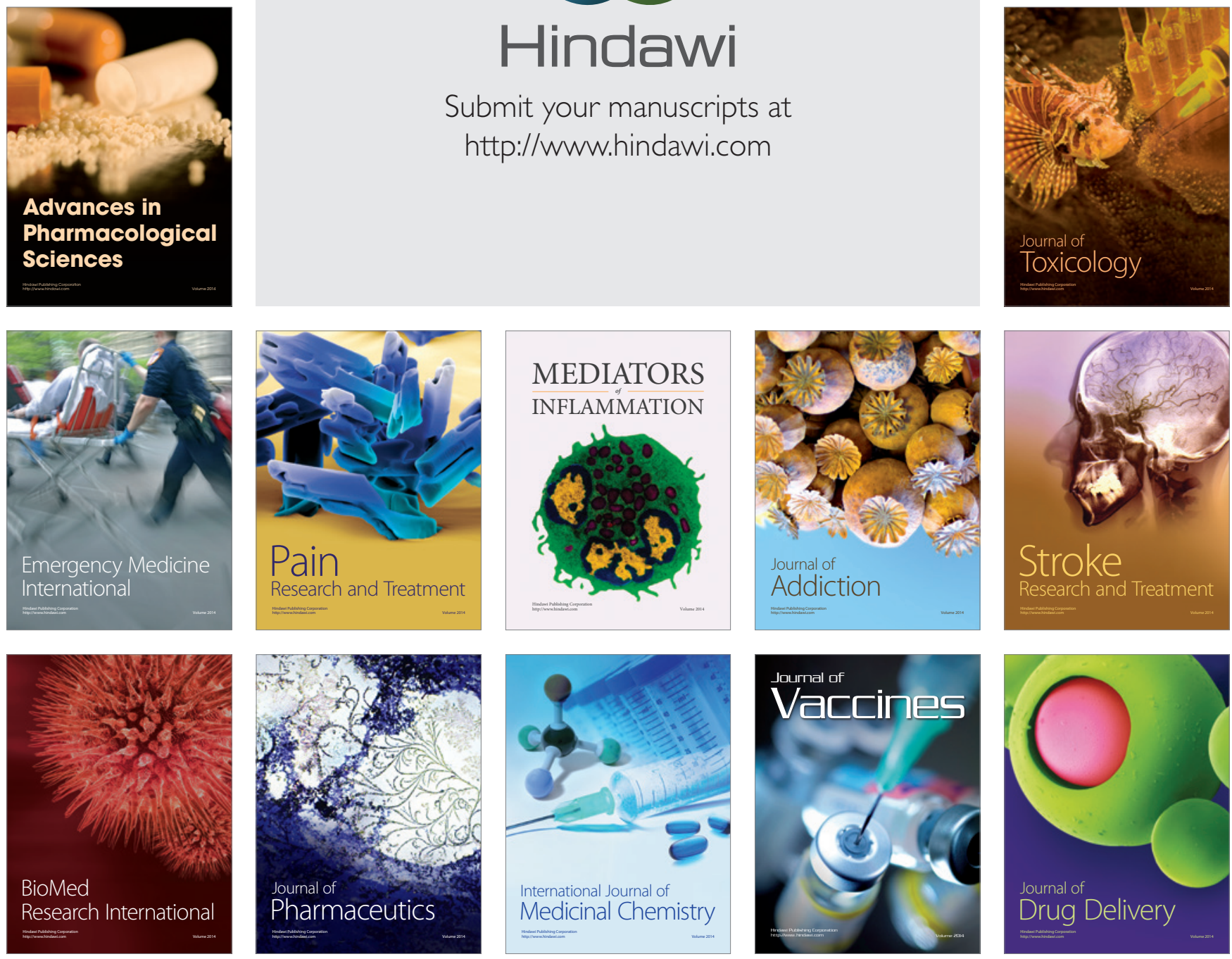